\title{
Measurements of wind turbulence parameters by a conically scanning coherent Doppler lidar in the atmospheric boundary layer
}

\author{
Igor N. Smalikho and Viktor A. Banakh \\ V. E. Zuev Institute of Atmospheric Optics SB RAS, Tomsk, Russia \\ Correspondence to: Igor N. Smalikho (smalikho@iao.ru )
}

Received: 1 May 2017 - Discussion started: 19 June 2017

Revised: 11 September 2017 - Accepted: 24 September 2017 - Published: 7 November 2017

\begin{abstract}
The method and results of lidar studies of spatiotemporal variability of wind turbulence in the atmospheric boundary layer are reported. The measurements were conducted by a Stream Line pulsed coherent Doppler lidar (PCDL) with the use of conical scanning by a probing beam around the vertical axis. Lidar data are used to estimate the kinetic energy of turbulence, turbulent energy dissipation rate, integral scale of turbulence, and momentum fluxes. The dissipation rate was determined from the azimuth structure function of radial velocity within the inertial subrange of turbulence. When estimating the kinetic energy of turbulence from lidar data, we took into account the averaging of radial velocity over the sensing volume. The integral scale of turbulence was determined on the assumption that the structure of random irregularities of the wind field is described by the von Kármán model. The domain of applicability of the used method and the accuracy of the estimation of turbulence parameters were determined. Turbulence parameters estimated from Stream Line lidar measurement data and from data of a sonic anemometer were compared.
\end{abstract}

\section{Introduction}

Pulsed coherent Doppler lidars (PCDLs) are applied in various fields of scientific research, in particular, to study dynamic processes in the atmosphere, aircraft wake vortices, and wind turbine wakes (Banakh and Smalikho, 2013). PCDLs are quite promising for obtaining reliable estimates of wind turbulence parameters from lidar measurements in the entire atmospheric boundary layer (Eberhard et al., 1989; Gal-Chen et al., 1992; Frehlich et al., 1998, 2006; Frehlich and Cornman, 2002; Davies et al., 2004; Smalikho et al.,
2005; Banta et al., 2006; O’Connor et al., 2010; Banakh and Smalikho, 2013; Sathe and Mann, 2013; Smalikho and Banakh, 2013; Smalikho et al., 2013; Sathe et al., 2015). For this purpose, different measurement geometries were proposed, and methods were developed for estimation of turbulence parameters, in particular, with allowance made for averaging of the radial velocity over the sensing volume and for the instrumental measurement error. Here, the radial velocity $V_{\mathrm{r}}$ is understood as a projection of the wind vector $\boldsymbol{V}=\left\{V_{z}, V_{x}, V_{y}\right\}\left(V_{z}\right.$ is the vertical component, $V_{x}$ and $V_{y}$ are the horizontal components) onto the axis of the probing beam at the point $\boldsymbol{r}=\{z, x, y\}=R \boldsymbol{S}$, where $R$ is the distance from the lidar, $S=\{\sin \varphi, \cos \varphi \cos \theta, \cos \varphi \sin \theta\}, \varphi$ is the elevation angle, and $\theta$ is the azimuth angle. Denoted are the average wind velocity and the wind direction angle as $U$ and $\theta_{V}$, respectively, and fluctuations of the vertical, longitudinal, and transverse wind components as $w, u$, and $v$.

The use of the conical scanning by the probing beam (when the elevation angle $\varphi$ is fixed during measurements, while the azimuth angle $\theta=\omega_{\mathrm{s}} t$ varies in time $t$ with the constant angular rate $\omega_{\mathrm{s}}$ ) allows reconstruction of not only the wind speed and direction, but also vertical profiles of different wind turbulence parameters from measurements by PCDL. It was shown by Eberhard et al. (1989) that the kinetic energy of turbulence $E=\left(\sigma_{w}^{2}+\sigma_{u}^{2}+\sigma_{v}^{2}\right) / 2$ can be determined from measurements by conically scanning PCDL at the elevation angle $\varphi=35.3^{\circ}$, where $\sigma_{w}^{2}=<w^{2}>, \sigma_{u}^{2}=<$ $u^{2}>, \sigma_{v}^{2}=<v^{2}>$, and the angular brackets denote the ensemble averaging. However, in the results for $E$, the effect of the averaging of the radial velocity over the sensing volume (see Eq. 6 in Smalikho and Banakh, 2013) was not taken into account. A method for reconstructing the vertical profiles of 
the fluxes of momentum $\langle u w>$ and $\langle v w>$ was also proposed by Eberhard et al. (1989).

Methods for determination of the turbulent energy dissipation rate $\varepsilon$ and the integral scale of turbulence $L_{V}=\int_{0}^{\infty} \mathrm{d} r B_{\|}(r) / \sigma_{\mathrm{r}}^{2}$, where $B_{\|}(r)$ is the longitudinal correlation function and $\sigma_{\mathrm{r}}^{2}=B_{\|}(0)$ is the variance of the radial wind velocity, from measurements by conically scanning PCDL have been proposed (Frehlich et al., 2006; Smalikho and Banakh, 2013; Smalikho et al., 2013). In this case, turbulence parameters are estimated through fitting of the theoretically calculated azimuth (transverse) structure function of the radial velocity measured by the lidar to the corresponding measured function on the assumption that turbulence is isotropic and its spatial structure is described by the von Kármán model (Vinnichenko et al., 1973). However, if the radius of the scanning cone base $R^{\prime}=R \cos \varphi$, where $R$ is the distance between the lidar and the center of the sensing volume, is comparable with or smaller than $L_{V}$, then the method of the azimuth structure function can give a large error in estimates of wind turbulence parameters (Smalikho and Banakh, 2013).

Pulsed coherent Doppler lidars capable of providing measured data with high spatial resolution (longitudinal size of the sensing volume can be around $30 \mathrm{~m}$ ), for example, Stream Line lidars (HALO Photonics) and Windcube lidars (Leosphere), are now widely used in practice. In this paper, for lidars of this type, we propose a method for the determination of wind turbulence parameters from measurements by conically scanning PCDLs, which removes the mentioned disadvantages of the earlier methods. With the use of the proposed method, we have obtained the time and height distributions of $E, \varepsilon, L_{V},<u w>$, and $<v w>$ in the atmospheric layer from 100 to $500 \mathrm{~m}$ in altitude from data of an atmospheric experiment with the Stream Line lidar. The accuracy of the obtained results is analyzed.

\section{Basic equations}

First, we describe the equations that will be used to develop the measurement strategy and the procedure of the estimation of wind turbulence parameters: $E, \varepsilon$, and $L_{V}$. Instantaneous values of components of the wind velocity vector are random functions of coordinates and time, that is, $\boldsymbol{V}=\boldsymbol{V}(\boldsymbol{r}, t)$. The radial velocity at a point moving in the cone base of conical scanning as the azimuth angle $\theta$ changes from 0 to $360^{\circ}$ (or in radians from 0 to $2 \pi$ ) can be represented in the form

$V_{\mathrm{r}}(\theta)=\boldsymbol{S}(\theta) \cdot \boldsymbol{V}\left(R \boldsymbol{S}(\theta), \theta / \omega_{\mathrm{s}}\right)$,

where $\varphi, R$, and $\omega_{\mathrm{s}}$ are constant parameters.

The turbulence is assumed to be stationary (for timescales no shorter than $1 \mathrm{~h}$ ) and horizontally homogeneous (within the scanning cone base). Because of anisotropy of wind turbulence, the variance of the radial velocity $\sigma_{\mathrm{r}}^{2}=<$
$\left[V_{\mathrm{r}}^{\prime}(\theta)\right]^{2}>$, where $V_{\mathrm{r}}^{\prime}=V_{\mathrm{r}}-<V_{\mathrm{r}}>$, is a function of the azimuth angle: $\sigma_{\mathrm{r}}^{2}=\sigma_{\mathrm{r}}^{2}(\theta)$. For the variance of the radial velocity averaged over the azimuth angles

$\bar{\sigma}_{\mathrm{r}}^{2}=(2 \pi)^{-1} \int_{0}^{2 \pi} \mathrm{d} \theta \sigma_{\mathrm{r}}^{2}(\theta)$,

and from Eqs. (1) and (2), after the corresponding ensemble averaging and integration over the angle $\theta$, we obtain the equation

$\bar{\sigma}_{\mathrm{r}}^{2}=(\sin \varphi)^{2} \sigma_{w}^{2}+(1 / 2)(\cos \varphi)^{2}\left(\sigma_{u}^{2}+\sigma_{v}^{2}\right)$.

From Eq. (3) at the angle $\varphi=\varphi_{\mathrm{E}}=\tan ^{-1}(1 / \sqrt{2}) \approx 35.3^{\circ}$, we can find a simple relation between the kinetic energy of turbulence $E$ and the variance $\bar{\sigma}_{\mathrm{r}}^{2}$ in the form (Eberhard et al., 1989)

$E=(3 / 2) \bar{\sigma}_{\mathrm{r}}^{2}$

Consider the azimuth structure function of the radial velocity $D_{\mathrm{r}}(\psi ; \theta)=<\left[V_{\mathrm{r}}^{\prime}(\theta+\psi)-V_{\mathrm{r}}^{\prime}(\theta)\right]^{2}>(\psi>0)$. For this function at $\psi \leq \pi / 2\left(90^{\circ}\right)$ and the fast movement of a point in a circle of the radius $R^{\prime}=R \cos \varphi$, when the condition $R^{\prime} \omega_{\mathrm{s}} \gg|\langle\boldsymbol{V}\rangle|$ is true, the transfer of turbulent inhomogeneities by the average flow can be neglected. Due to anisotropy of turbulence, the function $D_{\mathrm{r}}(\psi ; \theta)$, in the general case, depends on the angle $\theta$. By analogy with Eq. (2), we introduce the averaged structure function

$\bar{D}_{\mathrm{r}}(\psi)=(2 \pi-\psi)^{-1} \int_{0}^{2 \pi-\psi} \mathrm{d} \theta D_{\mathrm{r}}(\psi ; \theta)$.

Under the condition $\psi R^{\prime} \ll L_{V}$, due to the local isotropy of turbulence, $D_{\mathrm{r}}(\psi ; \theta)$ is independent of $\theta$, and $\bar{D}_{\mathrm{r}}(\psi)=D_{\mathrm{r}}(\psi)$. In addition, if the condition $R^{\prime}>L_{V}$ is also fulfilled, then, according to the Kolmogorov theory, $D_{\mathrm{r}}(\psi)$ is described by the equation (Kolmogorov, 1941)

$D_{\mathrm{r}}(\psi)=(4 / 3) C_{\mathrm{K}}\left(\varepsilon \psi R^{\prime}\right)^{2 / 3}$,

where $C_{\mathrm{K}} \approx 2$ is the Kolmogorov constant.

To find the relation between the structure function $\bar{D}_{\mathrm{r}}(\psi)$ and the integral scale $L_{V}$, it is necessary to know the equation for the correlation tensor of wind turbulence $B_{\alpha \beta}(\boldsymbol{r})=<$ $V_{\alpha}^{\prime}\left(\boldsymbol{r}_{0}+\boldsymbol{r}\right) V_{\beta}^{\prime}\left(\boldsymbol{r}_{0}\right)>\left(\alpha, \beta=z, x, y ; V^{\prime}=V-<V>\right)$, which can be readily found for the case of isotropic turbulence using an appropriate model for $B_{\|}(r)$. To find this relation, we assume that turbulence is isotropic, and within this assumption $\bar{D}_{\mathrm{r}}(\psi)=D_{\mathrm{r}}(\psi)$. Upon generalization of Eq. (19) given in Smalikho and Banakh (2013) for $\varphi=0^{\circ}$, for the case of an arbitrary elevation angle, we have derived the following equation: 


$$
\begin{aligned}
D_{\mathrm{r}}(\psi) & =4 \int_{0}^{\infty} \mathrm{d} \kappa S_{\|}(\kappa)\left[1-\mu_{1} \cos \left(2 \pi r^{\prime} \kappa\right)\right. \\
& \left.+\mu_{2} \pi r^{\prime} \kappa \sin \left(2 \pi r^{\prime} \kappa\right)\right],
\end{aligned}
$$

where $\mu_{1}=(\cos \varphi)^{2} \cos \psi+(\sin \varphi)^{2}$,

$\mu_{2}=(\cos \varphi)^{2}(1+\cos \psi) / 2+(\sin \varphi)^{2}$,

$r^{\prime}=R^{\prime} \sqrt{2(1-\cos \psi)}$,

and $S_{\|}(\kappa)=2 \int_{0}^{\infty} \mathrm{d} r B_{\|}(r) \cos (2 \pi \kappa r)$ is the longitudinal spatial spectrum of wind velocity fluctuations. If the condition $R^{\prime}=R \cos \varphi \gg L_{V}$ is fulfilled, in Eq. (7) we can set $\mu_{1}=\mu_{2}=1, r^{\prime}=y^{\prime}=\psi R^{\prime}$ (here, the angle $\psi$ is in radians), and then for any angles $\psi \leq 180^{\circ}$ the azimuth structure function $D_{\mathrm{r}}(\psi)$ coincides with the transverse structure function

$D_{\perp}\left(y^{\prime}\right)=4 \int_{0}^{\infty} \mathrm{d} \kappa S_{\perp}(\kappa)\left[1-\cos \left(2 \pi y^{\prime} \kappa\right)\right]$,

where $S_{\perp}(\kappa)=\left[S_{\|}(\kappa)-\kappa \mathrm{d} S_{\|}(\kappa) / \mathrm{d} \kappa\right] / 2$ is the transverse spectrum of wind velocity fluctuations (Lumley and Panofsky, 1964; Monin and Yaglom, 1971).

For the spectrum $S_{\|}(\kappa)$, we use the von Kármán model (Vinnichenko et al., 1973; Smalikho and Banakh, 2013):

$S_{\|}(\kappa)=2 \sigma_{\mathrm{r}}^{2} L_{V}\left[1+\left(C_{1} L_{V} \kappa\right)^{2}\right]^{-5 / 6}$,

where $C_{1}=8.4134$. For this model, the following relationship is true

$\sigma_{\mathrm{r}}^{2}=C_{2}\left(\varepsilon L_{\mathrm{V}}\right)^{2 / 3}$.

In Eq. (10) at $C_{\mathrm{K}}=2$, the coefficient $C_{2}=1.2717$ (Smalikho and Banakh, 2013).

Figure 1 shows the results of calculation of the normalized structure functions $D_{\mathrm{r}}(\psi) / \sigma_{\mathrm{r}}^{2}$ and $D_{\perp}\left(R^{\prime} \psi\right) / \sigma_{\mathrm{r}}^{2}$ at $\varphi=\varphi_{\mathrm{E}} \approx 35.26^{\circ}$ and different values of the ratio $R^{\prime} / L_{V}$. It can be seen that the higher the ratio, the smaller the difference between the functions. Calculations at $R^{\prime} / L_{V} \geq 4$ demonstrate the nearly complete coincidence of the structure functions described by Eqs. (7) and (8) for any angles $\psi \leq 180^{\circ}$. The nearly complete coincidence is also observed at $\psi \leq 9^{\circ}$ for any $R^{\prime} / L_{V} \geq 1 / 4$. At the same time, if the condition $R^{\prime} / L_{V} \ll \psi^{-1}$ is fulfilled, then, with allowance for Eq. (10), both structure functions $D_{\mathrm{r}}(\psi)$ and $D_{\perp}\left(R^{\prime} \psi\right)$ are described by Eq. (6).

We introduce the parameter $\gamma$ characterizing the degree of deviation of $D_{\perp}\left(R^{\prime} \psi\right)$ from $D_{\mathrm{r}}(\psi)$ as

$\gamma=\left\{L^{-1} \sum_{l=1}^{L}\left[D_{\mathrm{r}}(l \Delta \theta) / D_{\perp}\left(R^{\prime} l \Delta \theta\right)-1\right]^{2}\right\}^{1 / 2}$,

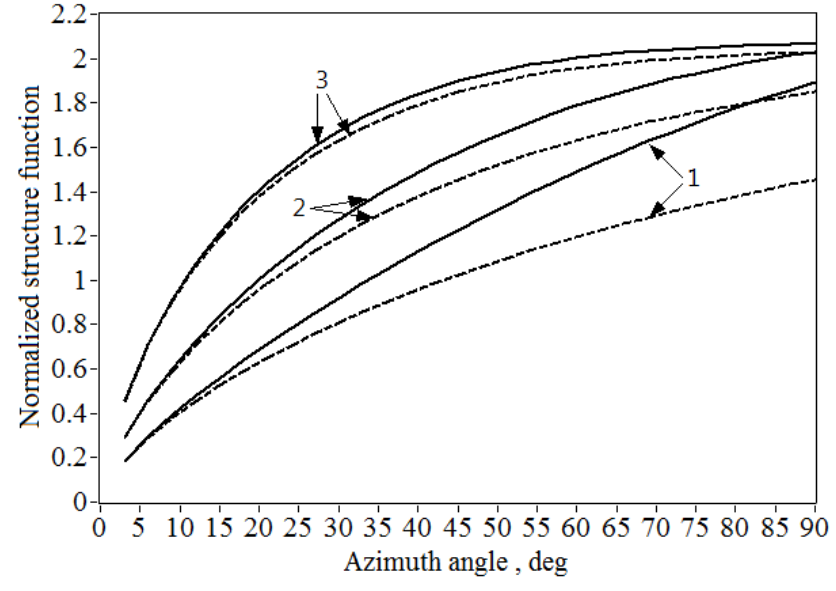

Figure 1. Normalized structure functions $D_{\mathrm{r}}(\psi) / \sigma_{\mathrm{r}}^{2}$ (solid curves) and $D_{\perp}\left(R^{\prime} \psi\right) / \sigma_{\mathrm{r}}^{2}$ (dashed curves) calculated, respectively, by Eqs. (7) and (8) with the use of the model (Eq. 9) at $R^{\prime} / L_{V}=0.5$ (curves 1), 1 (curves 2), and 2 (curves 3 ).

where $\Delta \theta=3^{\circ}$ and $L=30$. Using the data of Fig. 1, we have calculated the parameter $\gamma$ by this equation and obtained the following results: $\gamma=0.21$ at $R^{\prime} / L_{V}=0.5, \gamma=0.08$ at $R^{\prime} / L_{V}=1$, and $\gamma=0.02$ at $R^{\prime} / L_{V}=2$. It should be noted that, if we fit the function $D_{\perp}\left(R^{\prime} l \Delta \theta\right)$ with arbitrary values of $\varepsilon$ and $L_{V}$ by the least-squares method (see Eqs. 13-16 in Smalikho and Banakh, 2013) to the function $D_{\mathrm{r}}(l \Delta \theta)$ obtained at $R^{\prime} / L_{V}=0.5$, then we can attain a significant decrease in the parameter $\gamma$ (6 times in comparison with the above values), but the estimates of $L_{V}$ and $\sigma_{\mathrm{r}}^{2}$ exceed the true values of these parameters more than twice, although the error of $\varepsilon$ estimation by this method is about $15 \%$. Therefore, for these situations (when the ratio $R^{\prime} / L_{V}<1$ ), it is possible to obtain the more accurate result through direct determination of the variance $\sigma_{\mathrm{r}}^{2}$ and the dissipation rate $\varepsilon$ (the dissipation rate is determined from the azimuth structure function of the radial velocity within the inertial subrange of turbulence with the use of Eq. 6) and then calculation of the integral scale $L_{V}$ by Eq. (10).

\section{Measurement strategy and estimation of turbulence parameters}

To obtain the information about the kinetic energy, its dissipation rate, and the integral scale of turbulence from the same raw lidar data, it is proposed, according to the previous section, to use the conical scanning by the probing beam at the elevation angle $\varphi=\varphi_{\mathrm{E}} \approx 35.3^{\circ}$ in the experiment. During the measurements, the azimuth angle changes starting from $0^{\circ}$ with the constant angular rate $\omega_{\mathrm{s}}=2 \pi / T_{\text {scan }}$, where $T_{\text {scan }}$ is the time of one scan. As an angle of $360^{\circ}$ is achieved, the scanning in the opposite direction starts practically immediately. This cycle is repeated many times during the experiment. 
An array of estimates of the radial velocity $V_{\mathrm{L}}\left(\theta_{m}, R_{k}\right.$, $n$ ) is obtained from signals recorded by the PCDL-receiving system after the corresponding preprocessing (Banakh et al., 2015). Here, $\theta_{m}=m \Delta \theta$ is the azimuth angle; $m=0,1,2, \ldots$, $M-1 ; \Delta \theta$ is the azimuth resolution; $R_{k}=R_{0}+k \Delta R$ is the distance from the lidar to the center of the sensing volume; $R_{0}$ is the distance to the first usable range gate; $k=0,1$, $2, \ldots, K ; \Delta R$ is a range gate length; and $n=1,2,3, \ldots$, $N$ is the number of full conical scans. Uncertainty in the radial velocity measurement depends on the signal-to-noise ratio (SNR). At low SNR the probability of a "bad" estimate of the radial velocity randomly taking any values in the chosen receiver band (for example, $\pm 19.4 \mathrm{~m} \mathrm{~s}^{-1}$ for the Stream Line lidar), regardless of the true value of the velocity, can significantly differ from zero. To avoid the application of the data filtering procedure, the measured array $V_{\mathrm{L}}\left(\theta_{m}, R_{k}, n\right)$ must not contain "bad" estimates. Then, the lidar estimate of the radial velocity can be represented as (Frehlich and Cornman, 2002; Banakh and Smalikho, 2013)

$V_{\mathrm{L}}\left(\theta_{m}, R_{k}, n\right)=V_{\mathrm{a}}\left(\theta_{m}, R_{k}, n\right)+V_{\mathrm{e}}\left(\theta_{m}, R_{k}, n\right)$,

where $V_{\mathrm{a}}\left(\theta_{m}\right)$ is the radial velocity averaged over the sensing volume with the longitudinal dimension $\Delta z$ and the transverse dimension $\Delta y_{k}=\Delta \theta R_{k} \cos \varphi_{\mathrm{E}}$ (here, $\Delta \theta$ is in radians), and $V_{\mathrm{e}}\left(\theta_{m}\right)$ is the random instrumental error of estimation of the radial velocity having the following properties: < $V_{\mathrm{e}}>=<V_{\mathrm{a}} V_{\mathrm{e}}>=0$ and $<V_{\mathrm{e}}\left(\theta_{m}\right) V_{\mathrm{e}}\left(\theta_{l}\right)>=\sigma_{\mathrm{e}}^{2} \delta_{m-l}\left(\sigma_{\mathrm{e}}^{2}\right.$ is the variance of random error, $\delta_{m}$ is the Kronecker delta). For the conditions of stationary and homogeneous turbulence, the estimate is unbiased - that is, $<V_{\mathrm{L}}\left(\theta_{m}, R_{k}\right.$, $n)>=<V_{\mathrm{r}}\left(\theta_{m}, R_{k}\right)>$.

Lidars of Stream Line type, one of which is used in our experiments, are characterized by formation of a sensing volume of relatively small size, for example, with the longitudinal dimension $\Delta z=30 \mathrm{~m}$ (Pearson et al., 2009). When the conical scanning with $\varphi=\varphi_{\mathrm{E}}$ and $\Delta \theta=3^{\circ}$ is used, the transverse dimension of the sensing volume increases linearly from $8.5 \mathrm{~m}$ at $R_{k}=200$ to $42.8 \mathrm{~m}$ at $R_{k}=1 \mathrm{~km}$. It is important to take into account the effect from averaging of the radial velocity over the sensing volume not only when estimating the dissipation rate $\varepsilon$ within the inertial subrange of turbulence, but also when estimating the parameters $E$ and $L_{V}$, especially, when $L_{V}$ exceeds the size of the sensing volume insignificantly. Even at the high signal-to-noise ratio and the large number of probing pulses used for accumulation of lidar data, when the variance $\sigma_{\mathrm{e}}^{2}$ is extremely small, it is necessary to take into account the instrumental error of estimation of the radial velocity if turbulence is very weak (Frehlich et al., 2006).

After the corresponding manipulations, from Eq. (12), taking into account statistical properties of the random error $V_{\mathrm{e}}\left(\theta_{m}\right)$, we derived the following equations for the variance and the structure function of the lidar estimate of the radial velocity averaged over all azimuth angles $\theta_{m}$ :
$\bar{\sigma}_{\mathrm{L}}^{2}=\bar{\sigma}_{\mathrm{a}}^{2}+\sigma_{\mathrm{e}}^{2}$,

$\bar{D}_{\mathrm{L}}\left(\psi_{l}\right)=\bar{D}_{\mathrm{a}}\left(\psi_{l}\right)+2 \sigma_{\mathrm{e}}^{2}$,

where $\quad \bar{\sigma}_{\alpha}^{2}=M^{-1} \sum_{m=0}^{M-1} \sigma_{\alpha}^{2}\left(\theta_{m}\right) ; \quad \sigma_{\alpha}^{2}\left(\theta_{m}\right)=<\left[V_{\alpha}^{\prime}\left(\theta_{m}\right)\right]^{2}>$; $\bar{D}_{\alpha}\left(\psi_{l}\right)=(M-l)^{-1} \sum_{m=0}^{M-1-l} D_{\alpha}\left(\psi_{l}, \quad \theta_{m}\right) ; \quad D_{\alpha}\left(\psi_{l}, \quad \theta_{m}\right)=$ $<\left[V_{\alpha}^{\prime}\left(\theta_{m}+\psi_{l}\right)-V_{\alpha}^{\prime}\left(\theta_{m}\right)\right]^{2}>; \quad V_{\alpha}^{\prime}=V_{\alpha}-<V_{\mathrm{r}}>\quad$ and subscript $\alpha$ is $\mathbf{L}$ or a. In Eqs. (13) and (14) it is assumed that $\sigma_{\mathrm{e}}$ is independent of the azimuth angle $\theta_{m}$. The variance $\bar{\sigma}_{\mathrm{a}}^{2}$ can be represented as

$\bar{\sigma}_{\mathrm{a}}^{2}=\bar{\sigma}_{\mathrm{r}}^{2}-\bar{\sigma}_{\mathrm{t}}^{2}$,

where $\quad \bar{\sigma}_{\mathrm{t}}^{2}=M^{-1} \sum_{m=0}^{M-1} \sigma_{\mathrm{t}}^{2}\left(\theta_{m}\right) \quad$ and $\quad \sigma_{\mathrm{t}}^{2}\left(\theta_{m}\right)=\sigma_{\mathrm{r}}^{2}\left(\theta_{m}\right)-$ $\sigma_{\mathrm{a}}^{2}\left(\theta_{m}\right)$ is the turbulent broadening of the Doppler spectrum (Banakh and Smalikho, 2013).

Having specified the high resolution in the azimuth angle (large number $M$ ) and $\varphi=\varphi_{\mathrm{E}}$, from Eqs. (13)-(15) with allowance made for Eq. (4), we obtain the equation for the kinetic energy of turbulence in the form

$E=(3 / 2)\left[\bar{\sigma}_{\mathrm{L}}^{2}-\bar{D}_{\mathrm{L}}\left(\psi_{1}\right) / 2+G\right]$,

where $G=\bar{\sigma}_{\mathrm{t}}^{2}+\bar{D}_{\mathrm{a}}\left(\psi_{1}\right) / 2$. At $L_{V}>\max \left\{\Delta z, \Delta y_{k}\right\}$, the dimensions of the sensing volume do not exceed the lowfrequency boundary of the inertial subrange, for which turbulence is locally isotropic and, correspondingly, $G \sim \varepsilon^{2 / 3}$. If the condition $l \Delta y_{k} \ll L_{V}$ is additionally fulfilled, then for calculation of the turbulent broadening of the Doppler spectrum $\bar{\sigma}_{\mathrm{t}}^{2}=\sigma_{\mathrm{t}}^{2}$ and the structure function $\bar{D}_{\mathrm{a}}\left(\psi_{l}\right)=D_{\mathrm{a}}\left(\psi_{l}\right)$ we can use the two-dimensional spatial KolmogorovObukhov spectrum. For these conditions, the Gaussian temporal profile of the probing pulse, and the rectangular time window used for obtaining of Doppler spectra, we have derived the following equations (Banakh and Smalikho, 2013):

$\sigma_{\mathrm{t}}^{2}=\varepsilon^{2 / 3} F\left(\Delta y_{k}\right)$,

$D_{\mathrm{a}}\left(\psi_{l}\right)=\varepsilon^{2 / 3} A\left(l \Delta y_{k}\right)$.

In Eqs. (17) and (18)

$$
\begin{gathered}
F\left(\Delta y_{k}\right)=\int_{0}^{\infty} \mathrm{d} \kappa_{1} \int_{0}^{\infty} \mathrm{d} \kappa_{2} \Phi\left(\kappa_{1}, \kappa_{2}\right)\left[1-H_{\|}\left(\kappa_{1}\right) H_{\perp}\left(\kappa_{2}\right)\right], \\
A\left(l \Delta y_{k}\right)=2 \int_{0}^{\infty} \mathrm{d} \kappa_{1} \int_{0}^{\infty} \mathrm{d} \kappa_{2} \Phi\left(\kappa_{1}, \kappa_{2}\right) H_{\|}\left(\kappa_{1}\right) \\
H_{\perp}\left(\kappa_{2}\right)\left[1-\cos \left(2 \pi l \Delta y_{k} \kappa_{2}\right)\right],
\end{gathered}
$$

where $\Phi\left(\kappa_{1}, \kappa_{2}\right)=C_{3}\left(\kappa_{1}^{2}+\kappa_{2}^{2}\right)^{-4 / 3}\left[1+(8 / 3) \kappa_{2}^{2} /\left(\kappa_{1}^{2}+\kappa_{2}^{2}\right)\right]$; $C_{3}=2 C_{2} /\left(3 \pi C_{1}^{2 / 3}\right)=0.0652$; 
$H_{\|}\left(\kappa_{1}\right)=\left[\exp \left\{-\left(\pi \Delta p \kappa_{1}\right)^{2}\right\} \operatorname{sinc}\left(\pi \Delta R \kappa_{1}\right)\right]^{2}$ is the longitudinal transfer function of the low-frequency filter, and $H_{\perp}\left(\kappa_{2}\right)=\left[\operatorname{sinc}\left(\pi \Delta y_{k} \kappa_{2}\right)\right]^{2}$ is the transverse one; $\Delta p=c \sigma_{\mathrm{p}} / 2 ; c$ is the speed of light; $2 \sigma_{\mathrm{p}}$ is the duration of the probing pulse determined by the $e^{-1}$ power level to the right and to the left of the peak point, $\Delta R=c T_{\mathrm{W}} / 2$, where $T_{\mathrm{W}}$ is the temporal window width; and $\operatorname{sinc}(x)=\sin x / x$.

In Eq. (16), $\bar{\sigma}_{\mathrm{L}}^{2}$ and $\bar{D}_{\mathrm{L}}\left(\psi_{1}\right)$ are directly determined from experimental data. To take into account the term $G=\varepsilon^{2 / 3}\left[F\left(\Delta y_{k}\right)+A\left(\Delta y_{k}\right) / 2\right]$ in Eq. (16), it is necessary to have information about the dissipation rate $\varepsilon$. According to Eq. (14), the difference $\bar{D}_{\mathrm{L}}\left(\psi_{l}\right)-\bar{D}_{\mathrm{L}}\left(\psi_{1}\right)$ is equal to the difference $\bar{D}_{\mathrm{a}}\left(\psi_{l}\right)-\bar{D}_{\mathrm{a}}\left(\psi_{1}\right)$. Within the framework of the above conditions and according to Eq. (18), the latter is equal to $\varepsilon^{2 / 3}\left[A\left(l \Delta y_{k}\right)-A\left(\Delta y_{k}\right)\right]$. Then the dissipation rate can be determined as

$\varepsilon=\left[\frac{\bar{D}_{\mathrm{L}}\left(\psi_{l}\right)-\bar{D}_{\mathrm{L}}\left(\psi_{1}\right)}{A\left(l \Delta y_{k}\right)-A\left(\Delta y_{k}\right)}\right]^{3 / 2}$,

where the number $l>1$ should be so that, on the one hand, the consideration is within the inertial subrange and, on the other hand, the condition

$\left[\bar{D}_{\mathrm{L}}\left(\psi_{l}\right)-\bar{D}_{\mathrm{L}}\left(\psi_{1}\right)\right] \gg \bar{D}_{\mathrm{L}}\left(\psi_{1}\right) \sqrt{2 /(M N)}$

is fulfilled. This condition provides for the high accuracy of the estimation of the dissipation rate at the large numbers $M$ and $N$. In parallel, we can calculate the instrumental error of estimation of the radial velocity $\sigma_{\mathrm{e}}$ as

$$
\begin{aligned}
\sigma_{\mathrm{e}} & =\sqrt{\left[\bar{D}_{\mathrm{L}}\left(\psi_{1}\right)-\varepsilon^{2 / 3} A\left(\Delta y_{k}\right)\right] / 2} \\
& \equiv \sqrt{\frac{\bar{D}_{\mathrm{L}}\left(\psi_{1}\right) A\left(l \Delta y_{k}\right)-\bar{D}_{\mathrm{L}}\left(\psi_{l}\right) A\left(\Delta y_{k}\right)}{2\left[A\left(l \Delta y_{k}\right)-A\left(\Delta y_{k}\right)\right]}} .
\end{aligned}
$$

Using the lidar estimates of the kinetic energy $E$ (by Eq. 16) and the dissipation rate $\varepsilon$ from experimental data, we can determine the integral scale $L_{V}$ by Eqs. (4) and (10) as

$L_{V}=C_{4} E^{3 / 2} / \varepsilon$,

where $C_{4}=\left[2 /\left(3 C_{2}\right)\right]^{3 / 2}=0.3796$.

Taking into account that the elevation angle $\varphi=\varphi_{\mathrm{E}}=\tan ^{-1}(1 / \sqrt{2})$, we use the following equation (Eberhard et al., 1989) for determination of the momentum fluxes $\langle u w>$ and $\langle v w>$ :

$$
<u w>+j<v w>=\frac{3}{\sqrt{2}} \frac{1}{M} \sum_{m=0}^{M-1} \sigma_{\mathrm{L}}^{2}\left(\theta_{m}\right) \exp \left[j\left(\theta_{m}-\theta_{V}\right)\right],
$$

where $j=\sqrt{-1}$. Since the instrumental error of estimation of the radial velocity $\sigma_{\mathrm{e}}$ is independent of the azimuth angle $\theta_{m}$ and within the sensing volume, turbulence is locally isotropic (the condition $L_{V}>\max \left\{\Delta z, \Delta y_{k}\right\}$ is assumed to be true); that is, $\sigma_{\mathrm{t}}^{2}$ does not depend on $\theta_{m}$, and it is not necessary here to take into account the instrumental error and the effect from averaging of the radial velocity over the sensing volume. Indeed, as shown by Eberhard et al. (1989), in the case of a horizontally homogeneous turbulence statistics and very large $M$, Eq. (25) is exact if $\sigma_{\mathrm{L}}^{2}\left(\theta_{m}\right)$ is replaced by $\sigma_{\mathrm{r}}^{2}\left(\theta_{m}\right)$. On the other hand, $\sigma_{\mathrm{L}}^{2}\left(\theta_{m}\right)=\sigma_{\mathrm{r}}^{2}\left(\theta_{m}\right)-\sigma_{\mathrm{t}}^{2}+\sigma_{\mathrm{e}}^{2}$. Taking into account that $\sigma_{\mathrm{t}}^{2}$ and $\sigma_{\mathrm{e}}^{2}$ do not depend on $\theta_{m}$ and $\frac{1}{M} \sum_{m=0}^{M-1} \exp \left[j\left(\theta_{m}-\theta_{V}\right)\right]=0$, Eq. (25) can also be regarded as exact.

With increasing range $R_{k}=R_{0}+k \Delta R$, the measurement height $h_{k}=R_{k} \sin \varphi$ and the transverse dimension of the sensing volume $\Delta y_{k}=\Delta \theta R_{k} \cos \varphi$ increase linearly. Using Eqs. (19) and (20), we calculated $F\left(\Delta y_{k}\right)$ and $A\left(l \Delta y_{k}\right)$ by specifying the parameters of the lidar experiment conducted in 2016 (see Sect. 5) - that is, $\varphi=\varphi_{\mathrm{E}}=35.3^{\circ}, \Delta \theta=\pi / 60$ $\left(3^{\circ}\right), \Delta R=18 \mathrm{~m}$, and $\Delta p=15.3 \mathrm{~m}$. Without taking into account the spatial averaging of the radial velocity over the sensing volume, in Eq. (20) we set $H_{\|}\left(\kappa_{1}\right)=H_{\perp}\left(\kappa_{2}\right)=1$ and $A\left(l \Delta y_{k}\right)=A_{0}\left(l \Delta y_{k}\right)$. Then, after integrating over $\kappa_{1}$ and $\kappa_{2}$ in Eq. (20), we obtain the following equation: $A_{0}\left(l \Delta y_{k}\right)=2.667\left(l \Delta y_{k}\right)^{2 / 3}=(4 / 3) C_{\mathrm{K}}\left(l \Delta y_{k}\right)^{2 / 3}$. According to Fig. 1, the azimuth and transverse structure functions of the radial velocity completely coincide under the condition $l \Delta \theta \leq 9^{\circ}$. Therefore, we carried out calculations of $A\left(l \Delta y_{k}\right)$ at $l=1$ and $l=3$. To estimate the turbulent energy dissipation rate by Eq. (21), we set $l=3$. Denoted by $\varepsilon_{0}$ is the dissipation rate estimate obtained after the replacement of the difference $A\left(3 \Delta y_{k}\right)-A\left(\Delta y_{k}\right)$ by $A_{0}\left(3 \Delta y_{k}\right)-A_{0}\left(\Delta y_{k}\right)$ in Eq. (21). The ratio $\varepsilon / \varepsilon_{0}=\left\{\left[A_{0}\left(3 \Delta y_{k}\right)-A_{0}\left(\Delta y_{k}\right)\right] /\left[A\left(3 \Delta y_{k}\right)-A\left(\Delta y_{k}\right)\right]\right\}^{3 / 2}$ shows the degree of difference in the dissipation rate estimates with and without taking into account the averaging of the radial velocity over the sensing volume.

Figure 2 shows vertical profiles of $\Delta y_{k}, 3 \Delta y_{k}$, $F\left(\Delta y_{k}\right), \quad A\left(\Delta y_{k}\right), \quad A_{0}\left(\Delta y_{k}\right), \quad A\left(3 \Delta y_{k}\right), \quad A_{0}\left(3 \Delta y_{k}\right)$, $A\left(3 \Delta y_{k}\right)-A\left(\Delta y_{k}\right), \quad A_{0}\left(3 \Delta y_{k}\right)-A_{0}\left(\Delta y_{k}\right), \quad$ and $\varepsilon / \varepsilon_{0}$. The dashed line corresponds to the value of the longitudinal dimension of the sensing volume calculated as $\Delta z=\Delta R / \operatorname{erf}(\Delta R /(2 \Delta p))$, where $\operatorname{erf}(x)$ is the error function (Banakh and Smalikho, 2013). It is seen that with increasing height $h_{k}$ the transverse dimension of the sensing volume increases and at heights greater than $400 \mathrm{~m}$ it becomes larger than the longitudinal dimension $\Delta z$, which does not depend on the measurement height. The $F\left(\Delta y_{k}\right)$ takes values of $5.8 \mathrm{~m}^{2 / 3}$ at a height of $100 \mathrm{~m}$ and $8.3 \mathrm{~m}^{2 / 3}$ at a height of $500 \mathrm{~m}$ (see Fig. 2b). Figure $2 \mathrm{~b}$ also illustrates the effect of spatial averaging of the radial velocity on the azimuth (transverse) structure function of the radial velocity within the inertial subrange of turbulence (if the condition $3 \Delta y_{k} \ll L_{V}$ is satisfied). According to Fig. $2 \mathrm{~b}$, the ratio $A_{0}\left(\Delta y_{k}\right) / A\left(\Delta y_{k}\right)$ varies from 2.3 (at a height of $500 \mathrm{~m}$ ) to 4.2 (at a height of $100 \mathrm{~m}$ ) and the ratio $A_{0}\left(3 \Delta y_{k}\right) / A\left(3 \Delta y_{k}\right)$ varies from 1.4 (at a height of $500 \mathrm{~m}$ ) to 2 (at a height of $100 \mathrm{~m}$ ). As can be seen in 
Table 1. Average estimates of the kinetic energy and the integral scale of turbulence as functions of the number of scans used during the lidar measurements in the period from 12:00 to 18:00 LT on 22 July 2016 at a height of $200 \mathrm{~m}$.

\begin{tabular}{|c|c|c|c|c|c|c|}
\hline $\begin{array}{l}\text { Scan number (or measurement } \\
\text { duration in } \min \text { ) }\end{array}$ & 10 & 20 & 30 & 40 & 50 & 60 \\
\hline Kinetic energy of turbulence, $\left(\mathrm{m} \mathrm{s}^{-1}\right)^{2}$ & 1.71 & 1.84 & 1.88 & 1.91 & 1.92 & 1.93 \\
\hline Integral scale of turbulence, $\mathrm{m}$ & 208 & 231 & 239 & 244 & 247 & 249 \\
\hline
\end{tabular}
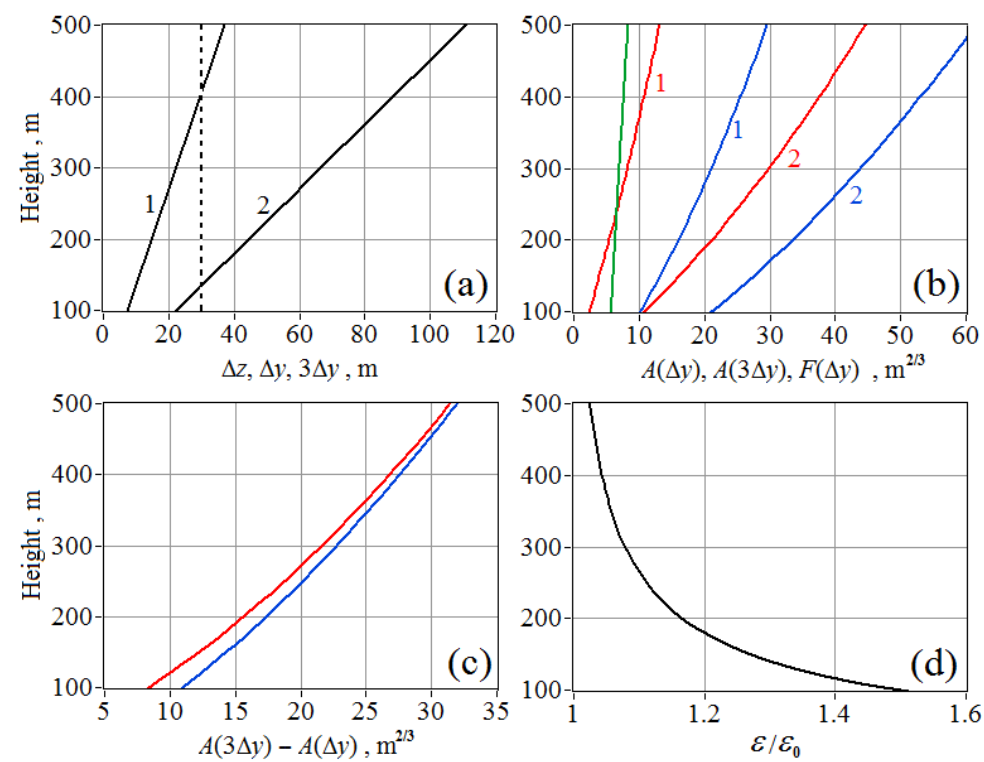

Figure 2. Vertical profiles of (a) longitudinal size of the sensing volume $\Delta z$ (dashed line), transverse size of the sensing volume $\Delta y_{k}$ (curve 1) and $3 \Delta y_{k}$ (curve 2); (b) $F\left(\Delta y_{k}\right)$ (green curve), $A\left(\Delta y_{k}\right)$ (red curve 1), $A_{0}\left(\Delta y_{k}\right)$ (blue curve 1), $A\left(3 \Delta y_{k}\right)$ (red curve 2), and $A_{0}\left(3 \Delta y_{k}\right)$ (blue curve 2); (c) differences $A\left(3 \Delta y_{k}\right)-A\left(\Delta y_{k}\right)$ (red curve) and $A_{0}\left(3 \Delta y_{k}\right)-A_{0}\left(\Delta y_{k}\right)$ (blue curve); (d) ratio $\varepsilon / \varepsilon_{0}$.

Fig. 2c, the difference between $A\left(3 \Delta y_{k}\right)-A\left(\Delta y_{k}\right)$ and $A_{0}\left(3 \Delta y_{k}\right)-A_{0}\left(\Delta y_{k}\right)$ is much smaller and, according to Fig. 2d, the estimate of the dissipation rate without taking into account the averaging of the radial velocity over the sensing volume is understated by 1.5 times for a height of $100 \mathrm{~m}$, and for heights above $375 \mathrm{~m}$, the underestimation does not exceed $5 \%$.

The practical implementation of the described method of the estimation of the wind turbulence parameters $\varepsilon, E, L_{V}$, $\langle u w\rangle$, and $\langle v w\rangle$ consists in the following. The obtained array $V_{\mathrm{L}}\left(\theta_{m}, R_{k}, n\right)$ for every height $h_{k}=R_{k} \sin \varphi_{\mathrm{E}}$ was used to determine the average wind vector $\langle\boldsymbol{V}\rangle$ (average wind velocity $U$ and wind direction angle $\theta_{V}$ ) with the use of the least-squares sine-wave fitting and the data of all $N$ scans. Then fluctuations of the radial velocity are calculated as $V_{\mathrm{L}}^{\prime}\left(\theta_{m}, R_{k}, n\right)=V_{\mathrm{L}}\left(\theta_{m}, R_{k}, n\right)-\boldsymbol{S}\left(\theta_{m}\right) \cdot\langle\boldsymbol{V}\rangle$, where $\boldsymbol{S}\left(\theta_{m}\right)=\left\{\sin \varphi_{\mathrm{E}}, \cos \varphi_{\mathrm{E}} \cos \theta_{m}, \cos \varphi_{\mathrm{E}} \sin \theta_{m}\right\}$ (in place of the array $\boldsymbol{S}\left(\theta_{m}\right) \cdot<\boldsymbol{V}>$, it is also possible to use directly the calculated values of $V_{\mathrm{L}}^{\prime}\left(\theta_{m}, R_{k}, n\right)=V_{\mathrm{L}}\left(\theta_{m}, R_{k}, n\right)-<$ $V_{\mathrm{L}}\left(\theta_{m}, R_{k}, n\right)>$ at the nonideal horizontal homogeneity of the average wind). Here and in Eqs. (13), (14), (16), (21)(23), and (25), the ensemble averaging $\langle X\rangle$ should be re- placed with the averaging over scans $N^{-1} \sum_{n=1}^{N} X_{n}$. The number of scans $N$ necessary for the averaging of data was determined experimentally (see Sect. 5).

To test the described method for measurement of the wind turbulence parameters, we have conducted experiments with the conically scanning Stream Line lidar (the main parameters of the lidar can be found in Table 1 in Banakh and Smalikho, 2016) and the sonic anemometer at a height of $43 \mathrm{~m}$ in 2014 and 2016.

\section{Experiment of 2014}

To study the feasibility of estimating the turbulence energy dissipation rate from PCDL data by the method described in Sect. 3 under various atmospheric conditions, we have conducted the 5-day experiment during 15-19 August of 2014 at the Basic Experimental Complex (BEC) of the Institute of Atmospheric Optics SB RAS. Experimental instrumentation included the Stream Line PCDL set at the central part of BEC mostly surrounded by forest and a sonic anemometer installed at the top of a tower (near BEC) at a height of $43 \mathrm{~m}$ 
from the ground. The separation between the lidar and the tower was $142 \mathrm{~m}$ (see Fig. 3).

Conical scanning by the probing beam with an angular rate of $5^{\circ} \mathrm{s}^{-1}$ (duration of one scan $T_{\text {scan }}=72 \mathrm{~s}$ ) at the elevation angle $\varphi=9^{\circ}$ was applied permanently during the experiment. For accumulation, an $N_{\mathrm{a}}=3000$ value of probing pulses were used. Since the pulse repetition frequency of the Stream Line lidar is $f_{\mathrm{p}}=15 \mathrm{kHz}$, the measurement for every azimuth scanning angle took $N_{\mathrm{a}} / f_{\mathrm{p}}=0.2 \mathrm{~s}$. In this case, for one scan we have $M=T_{\text {scan }} /\left(N_{\mathrm{a}} / f_{\mathrm{p}}\right)=360$ of such measurements with the resolution in the azimuth angle $\Delta \theta=1^{\circ}$. Since the lidar telescope is at a height of $1 \mathrm{~m}$ above the surface and the elevation angle is $9^{\circ}$, the probing pulse reaches the height of the sonic anemometer $(43 \mathrm{~m})$ at a distance of $270 \mathrm{~m}$. To increase the lidar signal-to-noise ratio at the height of $43 \mathrm{~m}$, the focus of the lidar beam was set to $300 \mathrm{~m}$. In Fig. 3, the blue circle shows the trajectory of the center of the sensing volume at a height of $43 \mathrm{~m}$ during the measurements.

From the array of radial velocities measured by the lidar in four full cycles of conical scanning $(N=4)$ for approximately $5 \mathrm{~min}$ (for this time at $R=270 \mathrm{~m}$ and $\varphi=9^{\circ}$, the sensing volume passes the distance $8 \pi R \cos \varphi$ equal to about $6.7 \mathrm{~km}$ ), we have calculated the values of the azimuth structure function $\bar{D}_{\mathrm{L}}\left(\psi_{1}\right)$ and $\bar{D}_{\mathrm{L}}\left(\psi_{l}\right)$. We obtained lidar estimates of the turbulent energy dissipation rate $\varepsilon_{\mathrm{L}}$ by Eq. (21) ( $\varepsilon$ should be replaced with $\varepsilon_{\mathrm{L}}$ ). To calculate the longitudinal structure functions $D_{\|}\left(r_{1}\right)$ and $D_{\|}\left(r_{2}\right)$ at separations of observation points $r_{1}=\Delta t_{1} U$ and $r_{2}=\Delta t_{2} U\left(r_{1}, r_{2}>0\right.$, $r_{2}>r_{1} ; \Delta t_{1}$ and $\Delta t_{2}$ are time separations), we used the array of longitudinal components of the wind vector measured by the sonic anemometer for the time $T=20 \mathrm{~min}$ (at a sampling frequency of $10 \mathrm{~Hz}$ ). For this time, at the average wind velocity $U=5 \mathrm{~m} \mathrm{~s}^{-1}$ typical of the surface layer, air masses move to a distance $U T=6 \mathrm{~km}$, which is quite comparable with the corresponding value for the lidar data (about $6.7 \mathrm{~km}$ ). We obtained estimates of the dissipation rate from the sonic anemometer data $\varepsilon_{\mathrm{S}}$ by the equation

$\varepsilon_{\mathrm{S}}=\left[\frac{D_{\|}\left(r_{2}\right)-D_{\|}\left(r_{1}\right)}{C_{\mathrm{K}} \cdot\left(r_{2}^{2 / 3}-r_{1}^{2 / 3}\right)}\right]^{3 / 2}$,

on the assumption that $l_{V} \ll r_{1}<r_{2} \leq r_{H}$, where $l_{V}$ is the inner scale of turbulence and $r_{H}$ is the scale of the lowfrequency boundary of the inertial subrange. Thus, the sample sizes for the lidar data and the sonic anemometer data are close, and the comparison of estimates of the dissipation rate $\varepsilon_{\mathrm{S}}$ and $\varepsilon_{\mathrm{L}}$ at properly specified $l, r_{1}, r_{2}$ and temporal synchronization of the results is quite justified.

According to the experimental data given in Byzova et al. (1989), the upper boundary of the inertial subrange $r_{H}$ at a height of $43 \mathrm{~m}$ takes values no smaller than $20 \mathrm{~m}$ at the neutral, unstable, and weak stable temperature stratification of the atmospheric boundary layer. In our case, $\Delta y_{k}=4.84 \mathrm{~m}$,

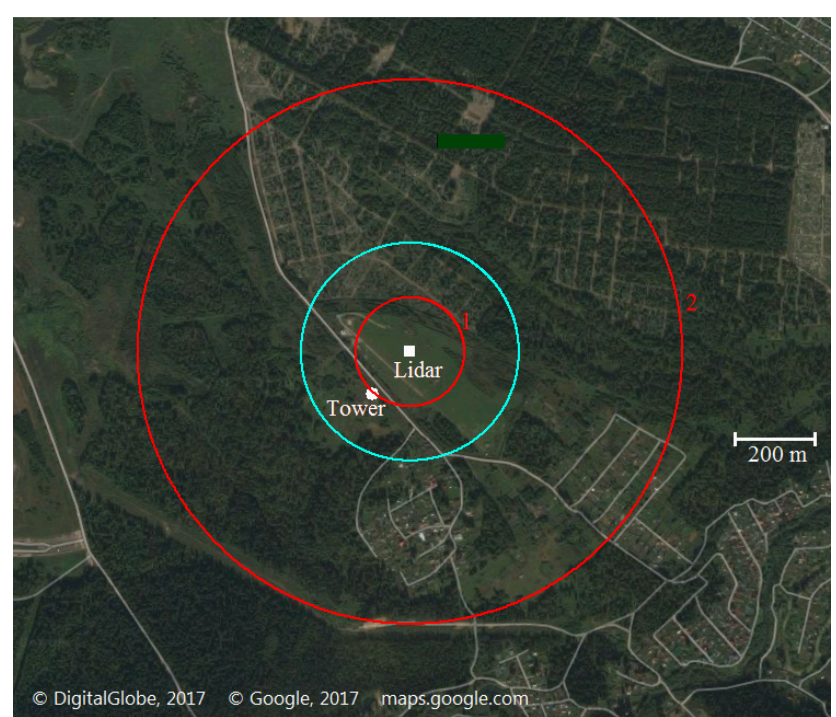

Figure 3. Map of the experimental site in 2014 and 2016. The blue circle shows the trajectory of the lidar sensing volume at a height of $43 \mathrm{~m}$ during the measurement at the elevation angle $\varphi=9^{\circ}$ in 2014 . Red circles 1 and 2 show the trajectories of the lidar sensing volume at heights of, respectively, 100 and $500 \mathrm{~m}$ during the measurement at $\varphi=35.3^{\circ}$ in 2016. Coordinates of the lidar point were $56^{\circ} 28^{\prime} 51.41^{\prime \prime} \mathrm{N}, 85^{\circ} 06^{\prime} 03.22^{\prime \prime} \mathrm{E}$.

and for $l=4$ the condition $l \Delta y \leq 20 \mathrm{~m}$ is true. In processing of the sonic anemometer data, we specified $r_{1}=5 \mathrm{~m}$ and $r_{2}=20 \mathrm{~m}$.

Lidar measurements were started at 18:00 LT (local time) on 15 August 2014 and finished at 14:30 LT on $19 \mathrm{Au}-$ gust 2014. Unfortunately, because of the weather conditions (low SNR) and some technical troubles, a portion (around $15 \%$ ) of the lidar data appeared to be unusable for the processing. Nevertheless, we succeeded in obtaining results under different atmospheric conditions for 5 days.

All the results of the estimation of the turbulent energy dissipation rate from the data measured by the sonic anemometer and the Stream Line lidar are shown in Fig. 4. One can see, in general, a rather good agreement between the results obtained from measurements by these devices. For calculation of the relative errors of the estimation of the dissipation rate $E_{\mathrm{S}}=\sqrt{<\left(\varepsilon_{\mathrm{S}} /<\varepsilon_{\mathrm{S}}>-1\right)^{2}>} \times 100 \%$ and $E_{\mathrm{L}}=\sqrt{<\left(\varepsilon_{\mathrm{L}} /<\varepsilon_{\mathrm{L}}>-1\right)^{2}>} \times 100 \%$, we used the data of Fig. 4 obtained from measurements under relatively steady conditions from 12:00 to 18:00 LT on 18 August. The errors appeared to be rather close: $E_{\mathrm{S}}=19 \%$ and $E_{\mathrm{L}}=20 \%$.

Using the data of Fig. 4, we have compared all estimates of the turbulent energy dissipation rate obtained from joint (simultaneous) measurements by the lidar and the sonic anemometer. The result of the comparison is shown in Fig. 5. Calculations of parameters characterizing discrepancies in the estimates of the dissipation rate $b_{\mathrm{LS}}=<\left(\varepsilon_{\mathrm{L}}-\varepsilon_{\mathrm{S}}\right) /\left[\left(\varepsilon_{\mathrm{L}}+\varepsilon_{\mathrm{S}}\right) / 2\right]>\times 100 \%$ and 


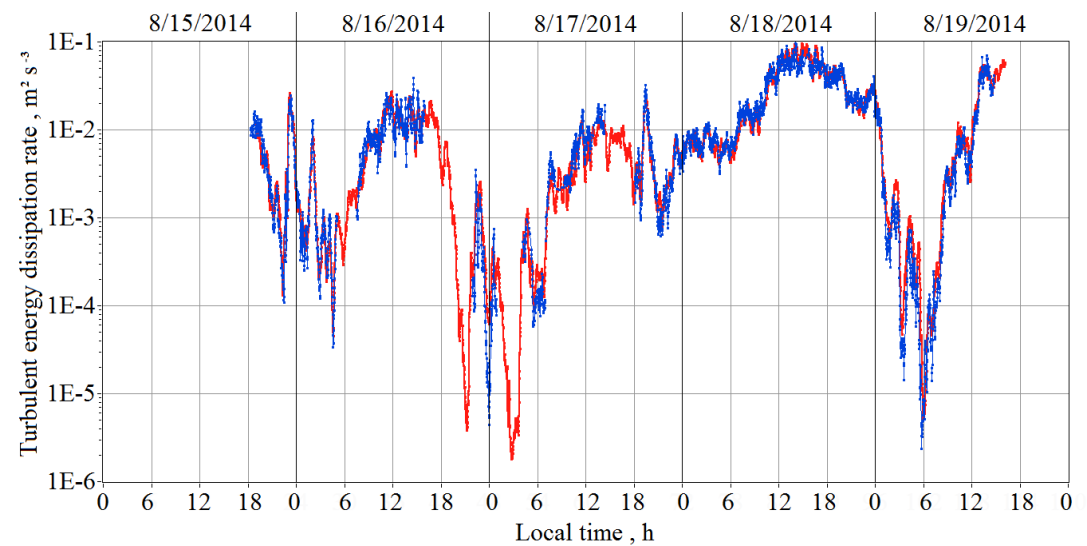

Figure 4. Time series of the turbulence energy dissipation rate obtained from measurements by the sonic anemometer (red curve) and the Stream Line lidar (blue curves) at a height of $43 \mathrm{~m}$.

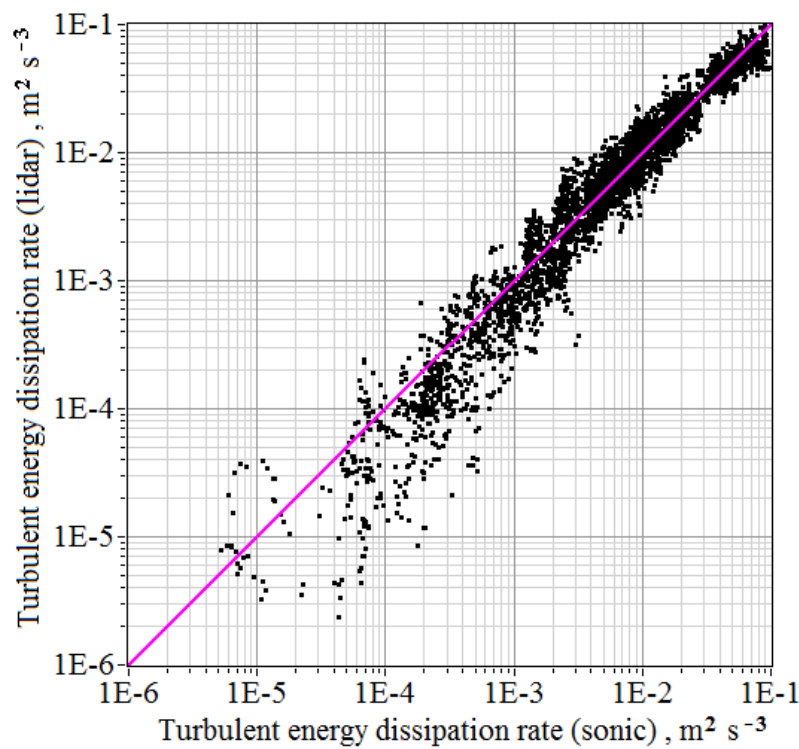

Figure 5. Comparison of estimates of the turbulence energy dissipation rate obtained from data of simultaneous measurements by the sonic anemometer and the Stream Line lidar. Time series of these estimates are shown in Fig. 4.

$\Delta_{\mathrm{LS}}=\sqrt{<\left(\varepsilon_{\mathrm{L}}-\varepsilon_{\mathrm{S}}\right)^{2} /\left[\left(\varepsilon_{\mathrm{L}}+\varepsilon_{\mathrm{S}}\right)^{2} / 4\right]>} \times 100 \%$ with the use of all points in Fig. 5 have shown that $b_{\mathrm{LS}}=-10 \%$ and $\Delta_{\mathrm{LS}}=45 \%$. Thus, the lidar estimate $\varepsilon_{\mathrm{L}}$ is, on average, $10 \%$ smaller than the estimate of the dissipation rate from the data of the sonic anemometer. If we assume that random errors of estimates from data of these devices are statistically independent and the variances of random errors are identical, the root-mean-square error of the estimate of the dissipation rate is about $30 \%$, which is 1.5 times higher than the value of $E_{\mathrm{L}}$ given above.

It can be easily seen from Fig. 5 that at $\varepsilon<10^{-3} \mathrm{~m}^{2} \mathrm{~s}^{-3}$ the lidar estimates of the dissipation rate $\varepsilon_{\mathrm{L}}$ are, on average, understated in comparison with the estimates $\varepsilon_{\mathrm{S}}$. According to Fig. 4, the estimates of the dissipation rate taking values smaller than $10^{-3} \mathrm{~m}^{2} \mathrm{~s}^{-3}$ were mostly obtained from nighttime measurements. As a rule, the temperature stratification is stable in nighttime, and then the upper boundary of the inertial subrange $r_{H}$ can be smaller than the spacing in observation points $4 \Delta y_{k}, r_{2} \sim 20 \mathrm{~m}$ used in Eqs. (21) and (26). In this case, estimates of the dissipation rate from the lidar and sonic anemometer data are understated, but the lidar estimate is understated to a greater extent because of the averaging of radial velocity over the sensing volume. Using the points of Fig. 5, whose coordinates satisfy the conditions $\varepsilon_{\mathrm{S}} \geq 10^{-3} \mathrm{~m}^{2} \mathrm{~s}^{-3}$ and $\varepsilon_{\mathrm{L}} \geq 10^{-3} \mathrm{~m}^{2} \mathrm{~s}^{-3}$, we have obtained $b_{\mathrm{LS}}=0$ and $\Delta_{\mathrm{LS}}=30 \%$. On the assumption of independent estimates from the data of the lidar and sonic anemometer and of the equal variances of estimates, the error of the lidar estimate of the dissipation rate, which can be calculated as $E_{\mathrm{L}}=\Delta_{\mathrm{LS}} / \sqrt{2}$, is equal to $21 \%$. Thus, for the conditions of moderate and strong turbulence, when $\varepsilon \geq 10^{-3} \mathrm{~m}^{2} \mathrm{~s}^{-3}$, the lidar estimate of the turbulence energy dissipation rate is unbiased, while the relative standard error of estimation is about $20 \%$.

\section{Experiment of 2016}

To test the method for determining the kinetic energy, its dissipation rate, the integral scale of turbulence, and momentum fluxes as described in Sect. 3, we have carried out the 5-day experiment from 19:00 LT on 20 July to 15:00 on 24 July 2016 at BEC. The Stream Line lidar was set exactly at the same place as in the experiment of 2014 (see Fig. 3). The weather was clear during these days. The presence of forest fires in the Tomsk region provided lidar measurements with rather high signal-to-noise ratios.

The Stream Line lidar operated continuously during the experiment. The focus of the lidar beam was set 
to $500 \mathrm{~m}$. The conical scanning with an angular rate of $6^{\circ} \mathrm{s}^{-1}$ (time of one full scan $T_{\text {scan }}=1 \mathrm{~min}$ ) at the elevation angle $\varphi=\varphi_{\mathrm{E}}=35.3^{\circ}$ was used. The number of probing pulses for data accumulation was $N_{\mathrm{a}}=7500$, which corresponded to the duration of measurement for every azimuth scanning angle $T_{\mathrm{a}}=0.5 \mathrm{~s}$. In this case, for one full scan we have $M=T_{\text {scan }} / T_{\mathrm{a}}=120$ such measurements with the resolution in the azimuth angle $\Delta \theta=3^{\circ}$. The range gate length $\Delta R$ was set equal to $18 \mathrm{~m}$ (vertical resolution $\Delta h=\Delta R \sin \varphi_{\mathrm{E}} \approx 10 \mathrm{~m}$ ).

In the processing of data of these measurements, we set the minimum useful range $R_{0}=171 \mathrm{~m}$, which corresponded to a minimum height of approximately $100 \mathrm{~m}$. Except for the period from 05:00 to 09:00 LT on 21 July 2016, the probability of "bad" lidar estimates of the radial velocity was zero for the ranges from $R_{0}$ to almost $900 \mathrm{~m}$. The maximum range $R_{K}$ was set equal to $873 \mathrm{~m}$, which corresponded to a height of about $500 \mathrm{~m}$. In this experiment, the linear velocity of horizontal motion of the sensing volume (along the base of the scanning cone) $V_{k}=2 \pi \cos \varphi_{\mathrm{E}} R_{k} / T_{\text {scan }}$ was $14.6 \mathrm{~m} \mathrm{~s}^{-1}$ for $R_{k}=R_{0}$ and $74.6 \mathrm{~m} \mathrm{~s}^{-1}$ for $R_{k}=R_{K}$. In this case, for $1 \mathrm{~min}$ the center of the sensing volume passed $876 \mathrm{~m}$ (at a height of $100 \mathrm{~m}$ ) and $4476 \mathrm{~m}$ (at a height of $500 \mathrm{~m}$ ). In Fig. 3, red circles 1 and 2 show the trajectories of the lidar sensing volume at heights of, respectively, 100 and $500 \mathrm{~m}$.

To obtain estimates of the wind turbulence parameters, raw data measured by some or other device for the time of 10 and $60 \mathrm{~min}$ are usually used. In our case, $T_{\text {scan }}=1 \mathrm{~min}$. This corresponds to the use of lidar data obtained for the number of full conical scans $N$ from 10 to 60 . To determine the optimal number $N$, we selected the lidar data measured at night and day on 22 July 2016 at a height of $200 \mathrm{~m}$ (1) from 01:00 to $07: 00$ and (2) from 12:00 to 18:00 LT. In these $6 \mathrm{~h}$ intervals, the horizontal wind speed averaged for $30 \mathrm{~min}$ varied from 11.5 to $13 \mathrm{~m} \mathrm{~s}^{-1}$ (night) and from 8 to $9.5 \mathrm{~m} \mathrm{~s}^{-1}$ (day).

Table 1 presents the averaged (for $6 \mathrm{~h}$ period) lidar estimates of the kinetic energy $E$ and the integral scale of turbulence $L_{V}$ obtained from measurements in daytime for different values of the scan number $N$. It should be noted that the average estimate of the dissipation rate $\varepsilon$ obtained from the same lidar data is independent of tabulated $N$ and equal to $4.1 \times 10^{-3} \mathrm{~m}^{2} \mathrm{~s}^{-3}$. It follows from Table 1 that, as the scan number increases, the estimates of the kinetic energy and the integral scale increase, and for $N>30$ (measurement time longer than $30 \mathrm{~min}$ ) the practically complete saturation takes place.

As for the estimates of the turbulence parameters from the nighttime measurement data in the considered period at a height of $200 \mathrm{~m}$, then the averaged (for $6 \mathrm{~h}$ period) estimate of the kinetic energy increases linearly with an increase in $N$ from $E=0.12\left(\mathrm{~m} \mathrm{~s}^{-1}\right)^{2}$ at $N=10$ to $E=0.24\left(\mathrm{~m} \mathrm{~s}^{-1}\right)^{2}$ at $N=60$ (2-fold increase). The similar increase is also observed for the estimate of the dissipation rate. At $N=30$, the average estimate $\varepsilon=5.5 \times 10^{-6} \mathrm{~m}^{2} \mathrm{~s}^{-3}$. The integral scale of turbulence determined by Eq. (24) has unrealistically high values $(\sim 4 \mathrm{~km})$, which indicates that the above method of lidar data processing is inapplicable to nighttime measurements above the atmospheric surface layer at stable temperature stratification. A possible reason is ignorance of the nonstationarity of the average wind, including mesoscale processes (for example, internal gravity waves), at very weak turbulence. Therefore, we restricted our consideration to the results of lidar measurements of turbulence only in the zone of intense mixing, which occurred in daytime. During the experiment, the intense mixing in the entire layer up to $500 \mathrm{~m}$ was observed approximately from 10:30 to 19:00 (21 July 2016), from 11:00 to 20:00 (22 July 2016), and from 11:30 to 18:00 LT (23 July 2016) (Smalikho and Banakh, 2017).

Figure 6 exemplifies the data of lidar measurements at different height. The value of $M^{-1} \sum_{m=0}^{M-1}\left[<V_{\mathrm{L}}\left(\theta_{m}\right.\right.$, $\left.\left.R_{k}\right)>-\boldsymbol{S}\left(\theta_{m}\right) \cdot<\boldsymbol{V}\left(h_{k}\right)>\right]^{2}$ is smaller than $\bar{\sigma}_{\mathrm{L}}^{2}$ by at least a factor of 10 . The blue curves in Fig. $6 \mathrm{~b}$ and $\mathrm{d}$ were obtained with the use of smoothing averaging over three points (azimuth angles). It can be seen that at negative values of the average radial velocity $<V_{\mathrm{L}}\left(\theta_{m}, R_{k}\right)>$ (or $\boldsymbol{S}\left(\theta_{m}\right) \cdot<$ $\boldsymbol{V}\left(h_{k}\right)>$ ) the variances of the lidar estimate of the radial velocity $\sigma_{\mathrm{L}}^{2}\left(\theta_{m}, h_{k}\right)$ mostly exceed the corresponding variances at the positive values of the average radial velocity. As a result, the estimates of the along-wind momentum flux $<u w>$ determined by Eq. (25) (real part) are negative, as expected (Lumley and Panofsky, 1964; Monin and Yaglom, 1971; Byzova et al., 1989; Eberhard et al., 1989; Sathe et al., 2015).

All our results of spatiotemporal visualization of the average wind, turbulence parameters, and instrumental error in estimation of the radial velocity from lidar measurements on 22 July 2016 in the period under consideration are shown in Fig. 7. Analogous results of the estimation of the turbulence parameters were also obtained from lidar measurements on 21 and 23 July 2016 in the above periods, but on 23 July the wind velocity $U$ was, on average, 1.8 times smaller than that on 22 July, while the kinetic energy $E$ was 2 to 2.5 times smaller, and the dissipation rate $\varepsilon$ was 2.5 to 4 times smaller (Smalikho and Banakh, 2017). At the same time, the estimates of the integral scale $L_{V}$ were, on average, close to each other (maximum deviation is around $20 \%$ ).

For illustration, Figs. 8 and 9 show, respectively, the time and height profiles of the wind turbulence parameters and the instrumental error in estimation of the radial velocity. The results presented for $\varepsilon, E, L_{V},\langle u w\rangle$, and $\langle v w\rangle$ do not contradict the theory of the atmospheric boundary layer and correspond to the known experimental data for similar atmospheric conditions (Lumley and Panofsky, 1964; Monin and Yaglom, 1971; Byzova et al., 1989). The instrumental error in estimation of the radial velocity $\sigma_{\mathrm{e}}$ depends mostly on the signal-to-noise ratio: the higher the SNR, the smaller $\sigma_{\mathrm{e}}$. Since the probing radiation was focused to a dis- 


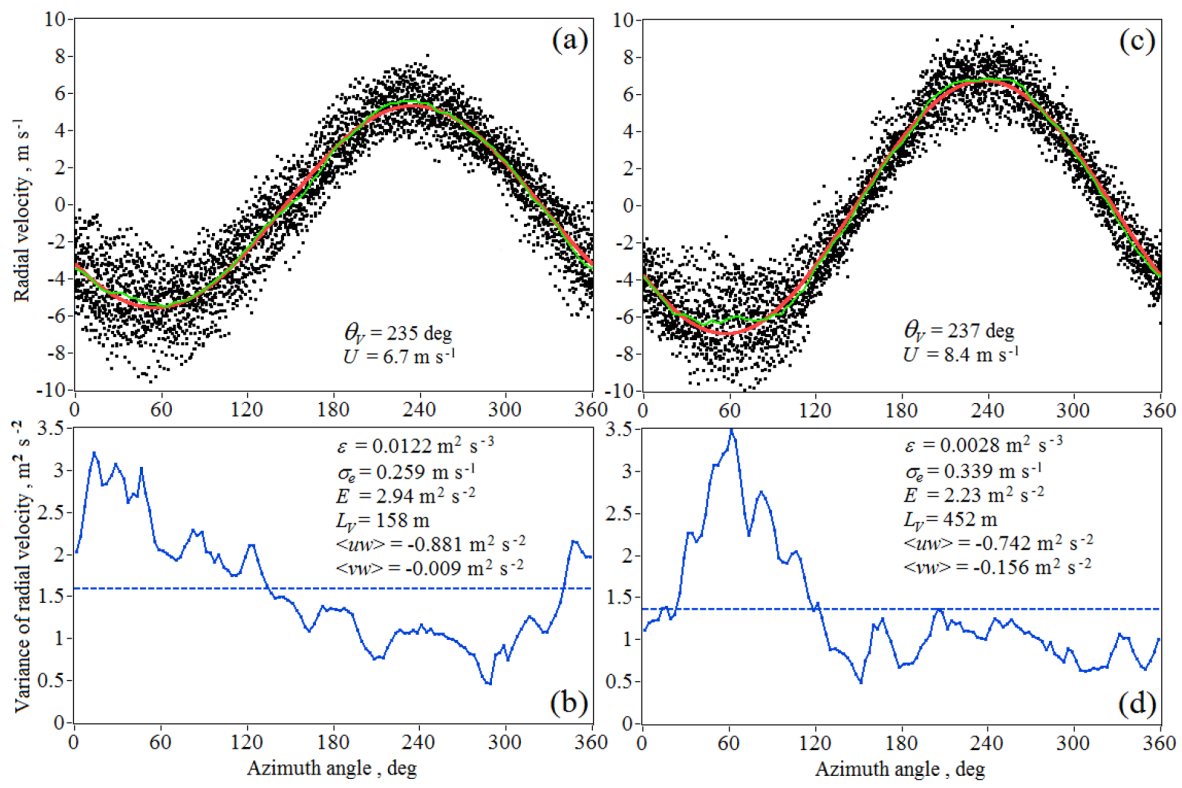

Figure 6. Single estimates of the radial velocity $V_{\mathrm{L}}\left(\theta_{m}, R_{k}, n\right)$ (dots); radial velocity averaged over 30 scans, $<V_{\mathrm{L}}\left(\theta_{m}, R_{k}\right)>$ (green curves); radial velocities as a result of sine-wave fitting, $V_{\mathrm{L}}\left(\theta_{m}, R_{k}\right)=\boldsymbol{S}\left(\theta_{m}\right) \cdot<\boldsymbol{V}\left(h_{k}\right)>$ (red curves) (a, c) and variances of the lidar estimate of the radial velocity $\sigma_{\mathrm{L}}^{2}\left(\theta_{m}, h_{k}\right)$ (blue curves) (b, d) as functions of the azimuth angle $\theta_{m}$ obtained from measurements by the Stream Line lidar on 22 July 2016 from 14:09 to 14:39 LT at the heights $h_{k}=R_{k} \sin \varphi_{\mathrm{E}}=109 \mathrm{~m}(\mathbf{a}, \mathbf{b})$ and $504 \mathrm{~m}$ (c, d). Dashed lines show variances averaged over the azimuth angle $\bar{\sigma}_{\mathrm{L}}^{2}$.

tance of $500 \mathrm{~m}, \sigma_{\mathrm{e}}$ took the smallest values in the layer of $200-300 \mathrm{~m}$. The error $\sigma_{\mathrm{e}}$ plays an important role in the fulfillment of the condition (Eq. 22) when turbulence is very weak. A necessary condition for obtaining the information about the turbulence energy dissipation rate $\varepsilon$ from lidar data with the use of Eq. (21) is the fulfillment of the inequality $R^{\prime} \psi_{l} \ll L_{V}$. In our case, for heights of 100,300 , and $500 \mathrm{~m}$ at $l=3$, the separation between the centers of the sensing volumes $R^{\prime} \psi_{3}$ is equal, respectively, to 22,67 , and $111 \mathrm{~m}$. According to the data of Figs. $7 \mathrm{~d}$ and $8 \mathrm{~d}$, this condition is true; that is, the dissipation rate is actually determined within the inertial subrange of turbulence.

Under the condition $L_{V} \gg \max \left\{\Delta z, \Delta y_{k}\right\}$, in accordance with Eq. (16), the estimate of the kinetic energy of turbulence can be represented as $E=(3 / 2)\left[\bar{\sigma}_{\mathrm{L}}^{2}-\sigma_{\mathrm{e}}^{2}+\sigma_{\mathrm{t}}^{2}\right]$, where the instrumental error in estimating the radial velocity $\sigma_{\mathrm{e}}$ and the turbulent broadening of the Doppler spectrum $\sigma_{\mathrm{t}}^{2}$ are determined using Eqs. (23) and (17), respectively. If $\sigma_{\mathrm{e}}^{2}$ and $\sigma_{t}^{2}$ are negligible, in comparison with the variance of the lidar estimate of the radial velocity $\bar{\sigma}_{\mathrm{L}}^{2}$, an estimate of the kinetic energy with a sufficiently high accuracy can be obtained using the equation: $E=(3 / 2) \bar{\sigma}_{\mathrm{L}}^{2}$. To study the effect of $\sigma_{\mathrm{e}}^{2}$ and $\sigma_{\mathrm{t}}^{2}$ on the estimation of the kinetic energy, we obtained vertical profiles of $E_{1}=(3 / 2) \bar{\sigma}_{\mathrm{L}}^{2}, E_{2}=(3 / 2)\left[\bar{\sigma}_{\mathrm{L}}^{2}-\sigma_{\mathrm{e}}^{2}\right]$, and $E_{3}=(3 / 2)\left[\bar{\sigma}_{\mathrm{L}}^{2}-\sigma_{\mathrm{e}}^{2}+\sigma_{t}^{2}\right]$. Four examples of such profiles are shown in Fig. 10. It can be seen that the allowance of the instrumental error $\sigma_{\mathrm{e}}$ is important in the layer above $400 \mathrm{~m}$, where the $\sigma_{\mathrm{e}}$ increases due to a decrease in the signal-to- noise ratio (see Figs. 7d, 8b and 9b). A comparison of the red and blue curves in Fig. 10 allows one to judge the effect of the allowance of the spatial averaging of the radial velocity over the sensing volume on the estimate of the turbulence kinetic energy. It follows from the data in Fig. 10 that the value $\left[\left(E_{3}-E_{2}\right) / E_{3}\right] \times 100 \%$ varies from 14 to $27 \%$ at a height of $100 \mathrm{~m}$ and from 10 to $16 \%$ at a height of $500 \mathrm{~m}$. If for estimating the integral scale of turbulence $L_{V}$ in Eq. (24), instead of $E \equiv E_{3}$, the $E_{2}$ is used, then the integral scale will be underestimated by $15-40 \%$.

The estimation of the integral scale of turbulence $L_{V}$ by Eq. (24) with the coefficient $C_{4}=0.38$ assumes that the spatial structure of wind turbulence is described by the von Kármán model. To clarify how close to reality this assumption is, we have compared the measured azimuth structure function $\bar{D}_{\mathrm{L}}\left(\psi_{l}\right)-2 \sigma_{\mathrm{e}}^{2}$ with the function $D_{\mathrm{a}}\left(\psi_{l}\right)=\varepsilon^{2 / 3} A\left(l \Delta y_{k}\right.$; $\left.L_{V}\right)$, where $A\left(l \Delta y_{k} ; L_{V}\right)$ is calculated by Eq. (20), which takes into account the integral scale of turbulence $L_{V}$ through replacement of $\Phi\left(\kappa_{1}, \kappa_{2}\right)$ with

$$
\begin{aligned}
\Phi\left(\kappa_{1}, \kappa_{2} ; L_{V}\right)= & \frac{1}{3 \pi} \frac{2 C_{1}^{2} C_{2} L_{V}^{8 / 3}}{\left[1+\left(C_{1} L_{V}\right)^{2}\left(\kappa_{1}^{2}+\kappa_{2}^{2}\right)\right]^{4 / 3}} \\
& {\left[1+\frac{8}{3} \cdot \frac{\left(C_{1} L_{V} \kappa_{2}\right)^{2}}{1+\left(C_{1} L_{V}\right)^{2}\left(\kappa_{1}^{2}+\kappa_{2}^{2}\right)}\right] . }
\end{aligned}
$$

Equation (27) was derived in Smalikho and Banakh (2013) with the use of the von Kármán model of isotropic turbulence. In calculations of $D_{\mathrm{a}}\left(\psi_{l}\right)=\varepsilon^{2 / 3} A\left(l \Delta y_{k} ; L_{V}\right)$, the ex- 

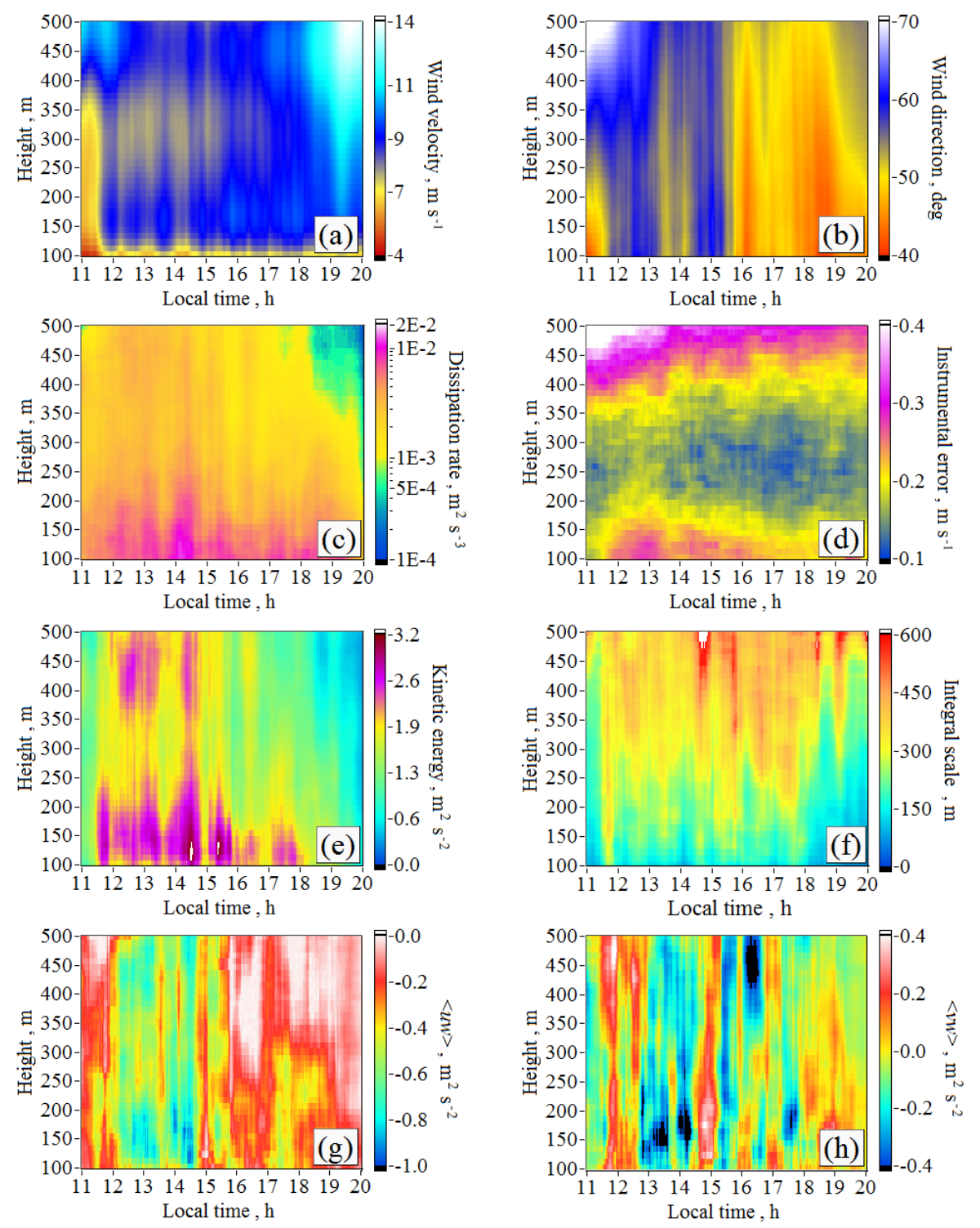

Figure 7. Spatiotemporal distributions of the average wind velocity $U$ (a), wind direction angle $\theta_{V}$ (b), turbulent energy dissipation rate $\varepsilon$ (c), instrumental error of estimation of the radial velocity $\sigma_{\mathrm{e}}(\mathbf{d})$, kinetic energy of turbulence $E$ (e), integral scale of turbulence $L_{V}$ (f), and momentum fluxes $\langle u w\rangle$ (g) and $\langle v w\rangle$ (h) obtained from measurements by the Stream Line lidar on 22 July 2016.

perimentally obtained values of $\varepsilon$ (from $\bar{D}_{\mathrm{L}}\left(\psi_{l}\right)$ within the inertial subrange of turbulence) and $L_{V}$ (with the use of Eq. 24) are used. We have also calculated the degree of deviation of the structure functions $\gamma$ by Eq. (11), where $D_{\mathrm{r}}(l \Delta \theta)$ and $D_{\perp}\left(R^{\prime} l \Delta \theta\right)$ were substituted with $\bar{D}_{\mathrm{L}}\left(\psi_{l}\right)-2 \sigma_{\mathrm{e}}^{2}$ and $D_{\mathrm{a}}\left(\psi_{l}\right)$, respectively.

Figure 11a depicts the spatiotemporal distribution of the parameter $\gamma$. According to this figure, the degree of deviation of the structure functions $\gamma$ varies from 0.014 to 0.22 (on average, about 0.1 ). The widest deviations are observed in the period from 12:30 to 14:30, when the lidar measurements were carried out under convective conditions of the atmospheric boundary layer. Figure 12 exemplifies the com- parison of the structure functions $\bar{D}_{\mathrm{L}}\left(\psi_{l}\right), \bar{D}_{\mathrm{L}}\left(\psi_{l}\right)-2 \sigma_{\mathrm{e}}^{2}$, and $D_{\mathrm{a}}\left(\psi_{l}\right)$. The last example demonstrates the importance of consideration of the instrumental error of the radial velocity in the estimation of wind turbulence parameters $\varepsilon, E$, and $L_{V}$. Figures $11 \mathrm{a}$ and 12 suggest that Eq. (24) with $C_{4}=0.38$ (von Kármán model) is applicable to the estimation of the integral scale $L_{V}$. Since turbulence is anisotropic, the estimated integral scale $L_{V}$ should be considered as the integral scale of turbulence averaged over the azimuth angles of conical scanning at an elevation angle of $35.3^{\circ}$.

To calculate the error of the lidar estimates of the dissipation rate, kinetic energy, and the integral scale of turbulence, we used the algorithm of numerical simulation, whose 

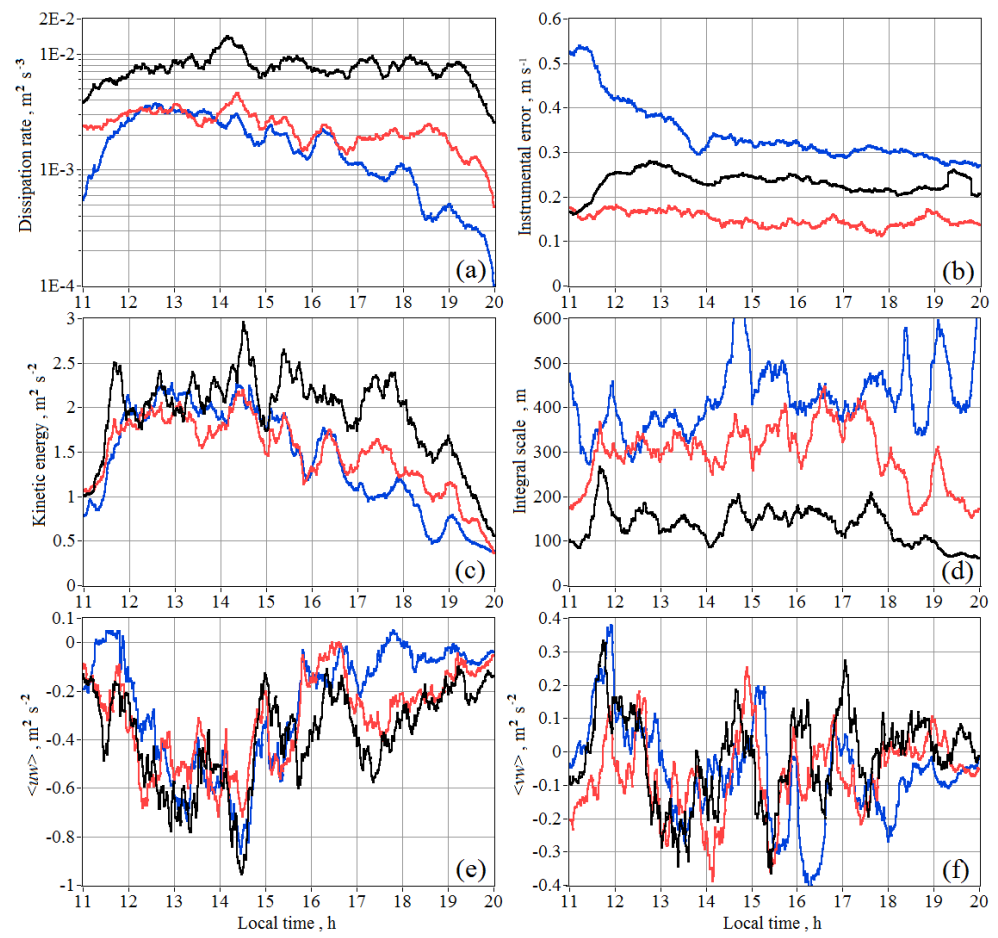

Figure 8. Temporal series of the turbulent energy dissipation rate $\varepsilon$ (a), instrumental error of radial velocity $\sigma_{\mathrm{e}}$ (b), kinetic energy of turbulence $E$ (c), integral scale of turbulence $L_{V}(\mathbf{d})$, and momentum fluxes $<u w>$ (e) and $<v w>$ (f) at heights of $100 \mathrm{~m}$ (black curves), $300 \mathrm{~m}$ (red curves), and $500 \mathrm{~m}$ (blue curves) taken from data of Fig. 7.
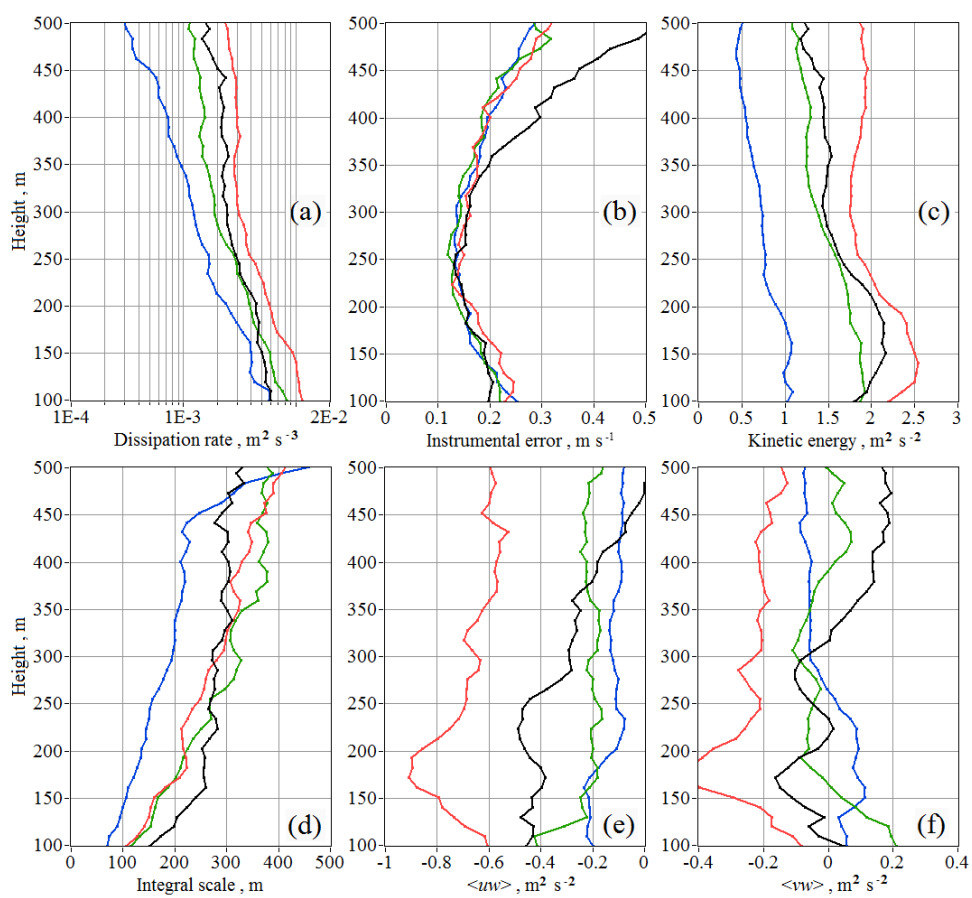

Figure 9. Vertical profiles of the turbulent energy dissipation rate $\varepsilon$ (a), instrumental error of radial velocity estimate $\sigma_{\mathrm{e}}$ (b), kinetic energy of turbulence $E$ (c), integral scale of turbulence $L_{V}(\mathbf{d})$, and momentum fluxes $<u w>$ (e) and $<v w>$ (f) at 11:30 LT (black curves), 14:00 LT (red curves), 17:00 LT (green curves), and 19:30 LT (blue curves) taken from the data of Fig. 7. 

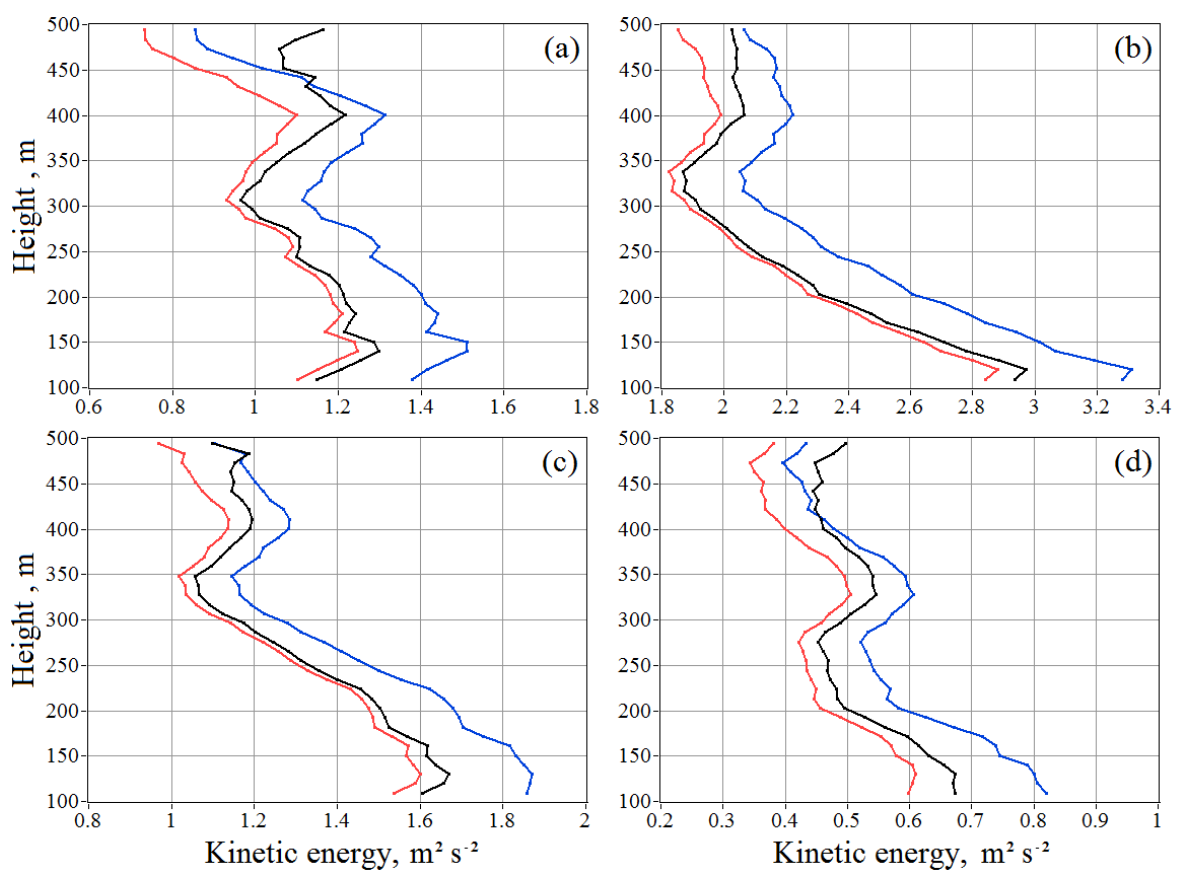

Figure 10. Vertical profiles of the turbulence kinetic energy estimates as $E_{1}=(3 / 2) \bar{\sigma}_{\mathrm{L}}^{2}$ (black curves), $E_{2}=(3 / 2)\left[\bar{\sigma}_{\mathrm{L}}^{2}-\sigma_{\mathrm{e}}^{2}\right]($ red curves), and $E_{3}=(3 / 2)\left[\bar{\sigma}_{\mathrm{L}}^{2}-\sigma_{\mathrm{e}}^{2}+\sigma_{\mathrm{t}}^{2}\right]$ (blue curves) obtained from measurements by Stream Line lidar on 29 July 2016 at 11:17 LT (a), 14:31 LT (b), 16:55 LT (c), and 19:47 LT (d).
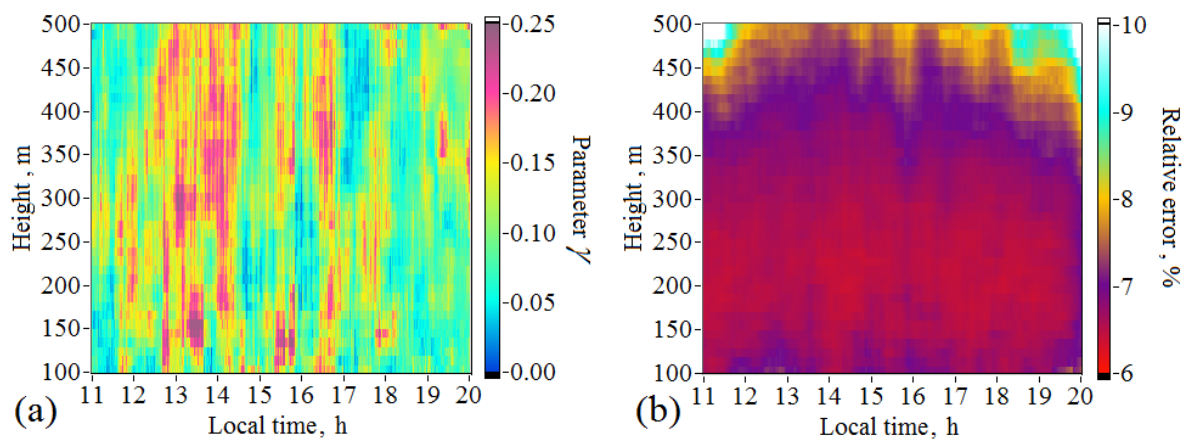

Figure 11. Time-height plots of the parameter $\gamma$ (a) and the relative error of estimation of the dissipation rate (b) obtained from measurements by the Stream Line lidar on 22 July 2016.

description can be found in papers of Smalikho and $\mathrm{Ba}-$ nakh (2013) and Smalikho et al. (2013). In the numerical simulation, we set the input parameters $U, \sigma_{\mathrm{e}}, \varepsilon, E$, and $L_{V}$ obtained from the lidar experiment. In addition, we assumed the stationarity and statistical homogeneity of the wind field and isotropy of turbulence. Figure $11 \mathrm{~b}$ shows the spatiotemporal distribution of the relative error of the lidar estimate of the turbulence energy dissipation rate. The error varies from 6.5 to $15 \%$. Figure 13 shows the time series and height profiles of the relative error of estimation of the dissipation rate. It can be seen that for the conditions of this experiment we have the rather high accuracy of determination of the dissipation rate from data of the conically scanning Stream Line lidar. Thus, in the layer of $100-350 \mathrm{~m}$, the relative error does not exceed $7.5 \%$. Worsening of the accuracy of the estimation of the dissipation rate with height is caused by an increase in the instrumental error $\sigma_{\mathrm{e}}$ and a decrease in the dissipation rate $\varepsilon$. It is shown in Sect. 4 that from lidar data measured for four scans it is possible to obtain the estimate of the dissipation rate with a relative error of $20 \%$. The results presented in this section were obtained from the data of 30 scans. In the case of stationary conditions, an increase in the scan number from 4 to 30 should lead to a decrease in the error from $20 \%$ to approximately $7 \%$ ( $\sqrt{30 / 4}$ times), which corresponds to the data of Figs. $11 \mathrm{~b}$ and 13 up to a height $\sim 350 \mathrm{~m}$.

According to the results of the numerical simulation for the experimental conditions considered in this section, the 

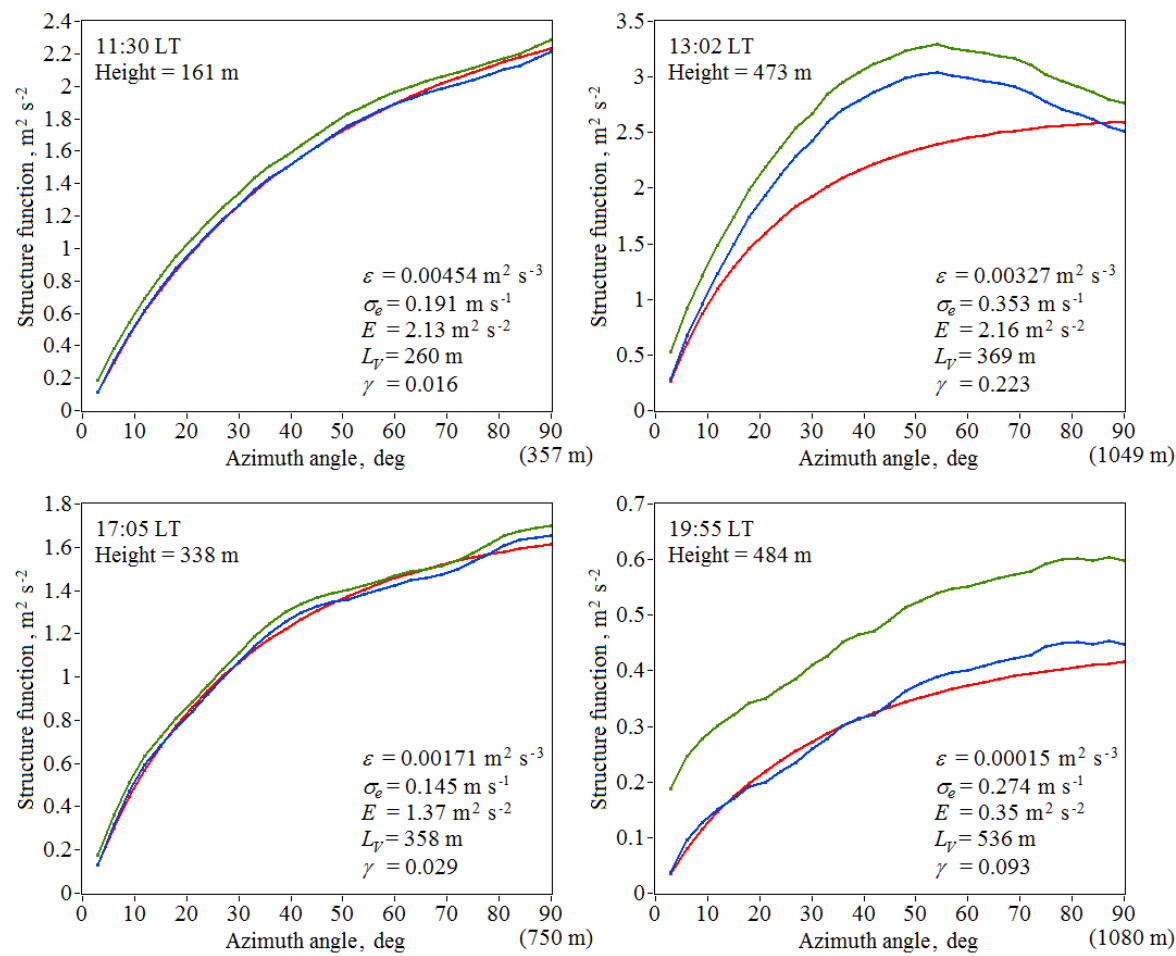

Figure 12. Examples of the azimuth structure functions $\bar{D}_{\mathrm{L}}\left(\psi_{l}\right)$ (green curves), $\bar{D}_{\mathrm{L}}\left(\psi_{l}\right)-2 \sigma_{\mathrm{e}}^{2}$ (blue curves), and $D_{\mathrm{a}}\left(\psi_{l}\right)$ (red curves) obtained from measurements by the Stream Line lidar on 22 July 2016. The $D_{\mathrm{a}}\left(\psi_{l}\right)$ functions were calculated by Eqs. (18), (20), and (27) with the use of experimental values of $\varepsilon$ and $L_{V}$. The arc length in the base of the scanning cone $y_{k}=\left(\pi / 180^{\circ}\right) \psi_{l} h_{k} / \tan \varphi_{\mathrm{E}}$ at $\psi_{l}=90^{\circ}$ is given in parenthesis.
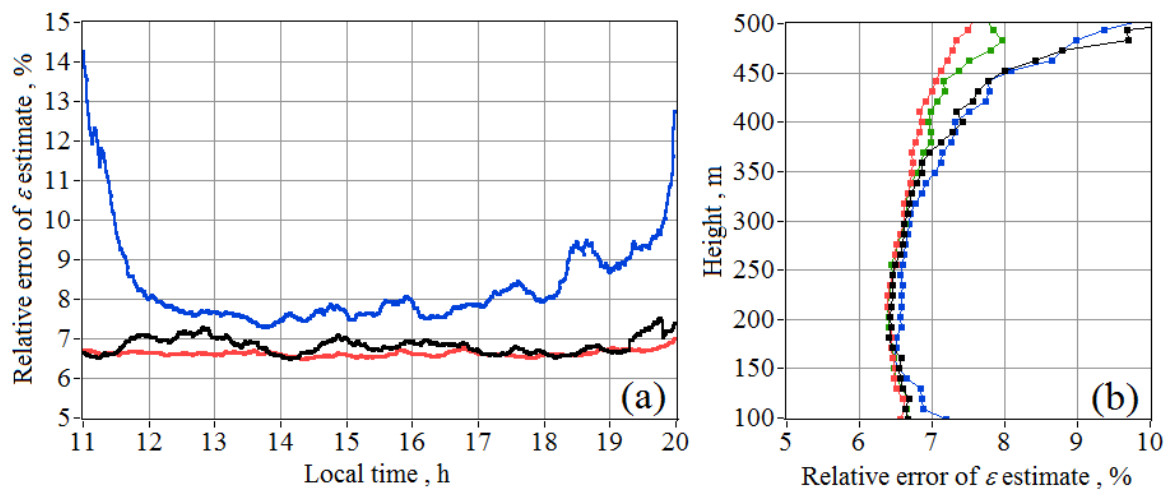

Figure 13. Time series of the relative error of estimation of the turbulence energy dissipation rate (a) at heights of $100 \mathrm{~m}$ (black curve), $300 \mathrm{~m}$ (red curve), and $500 \mathrm{~m}$ (blue curve), and height profiles of the relative error of estimation of the turbulence energy dissipation rate (b) at 11:30 LT (black squares), 14:00 LT (red squares), 17:00 LT (green squares), and 19:30 LT (blue squares) taken from the data of Fig. 11b.

relative error of the lidar estimate of the kinetic energy of turbulence varies insignificantly in the time and height ranges of Fig. $7 \mathrm{a}$ and averages about $10 \%$. At the same time, the relative error of estimation of the integral scale of turbulence varies from 16 to $20 \%$ as a function of height and time. A reliable way to study the capabilities of the considered method for the estimation of the turbulence parameters is by the com- parison of the results of simultaneous measurements by the lidar and the sonic anemometer at the same height.

Section 4 presents the results of simultaneous measurements of the dissipation rate $\varepsilon$ at a height of $43 \mathrm{~m}$ by the Stream Line lidar with conical scanning by the probing beam at the elevation angle $\varphi=9^{\circ}$ and the sonic anemometer installed at the tower (see Fig. 3). During the lidar measurements, whose results are presented above in Sect. 5, mea- 
surements by the sonic anemometer installed on the tower at a height of $43 \mathrm{~m}$ were carried out. Unfortunately, during these measurements the wind direction was such that the anemometer data were distorted due to wind flow around the tower. On 27 August 2016, we again conducted joint measurements by the Stream Line lidar (the elevation angle $\varphi$ was also set equal to $35.3^{\circ}$ ) and by the sonic anemometer, which measured raw data along the wind without distortions for $24 \mathrm{~h}$. Since the minimum distance of measurement by the Stream Line lidar is $120-150 \mathrm{~m}$, it was impossible to conduct lidar measurements at the anemometer height of $43 \mathrm{~m}$ at this elevation angle. Taking into account that the kinetic energy $E$ varies more smoothly with height in comparison with other turbulent parameters $\varepsilon$ and $L_{V}$, we have compared the diurnal profiles of the kinetic energy obtained from joint measurements by the Stream Line lidar at a height of $100 \mathrm{~m}$ and by the sonic anemometer at a height of $43 \mathrm{~m}$. The result of the comparison is shown in Fig. 14. Taking into account the difference in the measurement heights, we can say that a rather good agreement is observed between the time series of the kinetic energy of turbulence obtained from measurements by the different devices.

\section{Conclusions}

Thus, in this paper we have proposed a relatively simple method for the determination of the turbulence energy dissipation rate, kinetic energy, and integral scale of turbulence from measurements by conically scanning PCDL. The method is applicable in the case where the longitudinal and transverse dimensions of the sensing volume do not exceed the integral scale of turbulence. Since the dissipation rate is estimated from the azimuth structure function within the inertial subrange of turbulence, it is sufficient to calculate the function $A\left(l \Delta y_{k}\right)$ for different heights $h_{k}$ by Eq. (20), and then with Eq. (21) it is possible to retrieve the vertical profiles $\varepsilon\left(h_{k}\right)$. In the estimation of the kinetic energy of turbulence, the spatial averaging of the radial velocity over the sensing volume is taken into account. For this purpose, it is necessary to calculate the function $F\left(\Delta y_{k}\right)$ by Eq. (19) and to use Eq. (16). Then, the integral scale of turbulence is determined with Eq. (24). In contrast to the approach described by Frehlich et al. (2006) and Smalikho and Banakh (2013), in this method there is no need to calculate the azimuth structure function of the radial velocity averaged over the sensing volume with the use of the spectrum model in the form of Eq. (27) and to apply the procedure of the least-squares fitting of the calculated function to the measured one. As is shown in Sect. 2, this fitting in some cases can lead to the overestimation of the integral scale of turbulence. We have seen this when applying this fitting to the lidar data of the experiment (measurements in the daytime) described in Sect. 5. As a result, we have obtained unrealistically high values for the estimates of the integral scale of turbulence at low heights

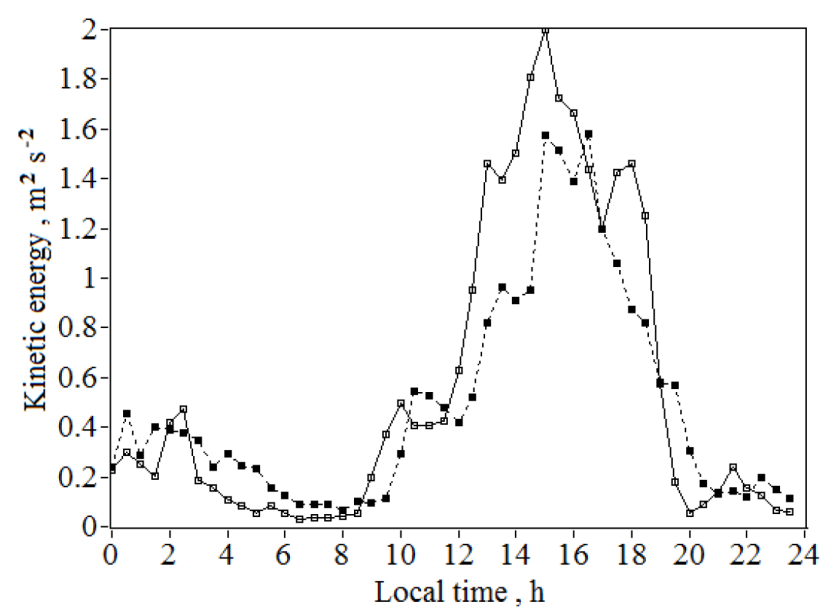

Figure 14. Diurnal profiles of the kinetic energy of turbulence obtained from simultaneous measurements by the sonic anemometer at a height of $43 \mathrm{~m}$ (solid curve) and the Stream Line lidar at a height of $100 \mathrm{~m}$ (dashed curve) on 27 August 2016.

of $100-200 \mathrm{~m}$. Sometimes such estimates exceed $1 \mathrm{~km}$, in contrast to results shown in Figs. 7f, 8d, and 9d.

The comparison of measurements of the turbulence energy dissipation rate by the Stream Line lidar with the method described in Sect. 3 and the data measured by the sonic anemometer has demonstrated a good agreement. The data of the lidar experiment of 2016 have been used to obtain the spatiotemporal distributions of different wind turbulence parameters with a height resolution of $10 \mathrm{~m}$ and a time resolution of $30 \mathrm{~min}$. The lidar estimates of turbulence have been analyzed. It has been shown that the use of conical scanning during measurements by PCDL and the method for processing of lidar data proposed in this paper allows obtaining the information about wind turbulence in the atmospheric mixing layer with a rather high accuracy. However, as shown by the lidar experiment conducted under stable temperature stratification outside the layer of intensive turbulent mixing (Smalikho and Banakh, 2017), this method is not applicable and, consequently, further investigations and development of new approaches are needed.

Data availability. All the data presented in this study are available from the authors upon request. 


\section{Appendix A: List of symbols}

\begin{tabular}{|c|c|}
\hline$B_{\|}(r)$ & Longitudinal correlation function of wind velocity \\
\hline$c$ & Speed of light \\
\hline$C_{\mathrm{K}} \approx 2$ & Kolmogorov constant \\
\hline \multicolumn{2}{|l|}{$C_{1}=8.4134$} \\
\hline \multicolumn{2}{|l|}{$C_{2}=1.2717$} \\
\hline \multicolumn{2}{|l|}{$C_{3}=0.0652$} \\
\hline \multicolumn{2}{|l|}{$C_{4}=0.3796$} \\
\hline$D_{\mathrm{r}}(\psi ; \theta)$ & Azimuth structure function of the radial velocity \\
\hline$D_{\mathrm{r}}(\psi)$ & Azimuth structure function of the radial velocity for isotropic turbulence \\
\hline $\bar{D}_{\mathrm{r}}(\psi)$ & Averaged azimuth structure function of the radial velocity (Eq. 5) \\
\hline $\bar{D}_{\mathrm{L}}\left(\psi_{l}\right)$ & Azimuth structure function of the lidar estimate of the radial velocity \\
\hline $\bar{D}_{\mathrm{a}}\left(\psi_{l}\right)$ & Azimuth structure function of the radial velocity averaged over the sensing volume \\
\hline$D_{\perp}\left(y^{\prime}\right)$ & Transverse structure function of wind velocity \\
\hline$E=\left(\sigma_{w}^{2}+\sigma_{u}^{2}+\sigma_{v}^{2}\right) / 2$ & Kinetic energy of turbulence \\
\hline$f_{\mathrm{p}}$ & Pulse repetition frequency \\
\hline$L_{V}$ & Integral scale of turbulence \\
\hline$l_{V}$ & Inner scale of turbulence \\
\hline$N$ & Number of conical scans \\
\hline$N_{\mathrm{a}}$ & Number of probing pulses used for the accumulation \\
\hline$R$ & Range (distance from lidar) \\
\hline$R_{0}$ & Minimum range \\
\hline$R^{\prime}=R \cos \varphi$ & Radius of the circle along which the sensing volume moves during the conical scanning \\
\hline$r_{H}$ & Scale of the low-frequency boundary of the inertial subrange \\
\hline$S_{\|}(\kappa)$ & Longitudinal spatial spectrum of wind velocity fluctuations \\
\hline$S_{\perp}(\kappa)$ & Transverse spatial spectrum of wind velocity fluctuations \\
\hline SNR & Signal-to-noise ratio \\
\hline$T_{\text {scan }}$ & Duration of one conical scan \\
\hline$T_{\mathrm{W}}$ & Temporal window width \\
\hline$u$ & Fluctuations of longitudinal wind component \\
\hline$U$ & Average wind velocity \\
\hline$<u w>$ & Along-wind momentum flux \\
\hline$v$ & Fluctuations of transverse wind component \\
\hline $\boldsymbol{V}=\left\{V_{z}, V_{x}, V_{y}\right\}$ & Wind vector, where $V_{z}$ is the vertical component, and $V_{x}$ and $V_{y}$ are the horizontal components \\
\hline$V_{\mathrm{a}}$ & Radial velocity averaged over the sensing volume \\
\hline$V_{\mathrm{e}}$ & Random instrumental error of unbiased estimate of the radial velocity \\
\hline$V_{\mathrm{L}}$ & Lidar estimate of the radial velocity \\
\hline$V_{\mathrm{r}}$ & Radial velocity \\
\hline$<v w>$ & Cross-wind component of momentum flux \\
\hline$w$ & Fluctuations of vertical wind component \\
\hline$\gamma$ & Parameter characterizing the degree of deviation of structure functions \\
\hline$\Delta h=\Delta R \sin \varphi_{\mathrm{E}}$ & Vertical resolution \\
\hline$\Delta R$ & Range gate length \\
\hline$\Delta y_{k}=\Delta \theta R_{k} \cos \varphi$ & Transverse size of the sensing volume at distance $R_{k}$ from the lidar \\
\hline$\Delta z$ & Longitudinal size of the sensing volume \\
\hline$\Delta \theta$ & Azimuth angle resolution \\
\hline$\varepsilon$ & Turbulent energy dissipation rate \\
\hline$\varepsilon_{\mathrm{L}}$ & Estimate of the turbulent energy dissipation rate from measurement by lidar \\
\hline$\varepsilon_{\mathrm{S}}$ & Estimate of the turbulent energy dissipation rate from measurement by sonic anemometer \\
\hline$\theta$ & Azimuth angle \\
\hline$\theta_{V}$ & Average wind direction angle \\
\hline$\sigma_{\mathrm{a}}^{2}=<V_{\mathrm{a}}^{2}>-<V_{\mathrm{a}}>^{2}$ & Variance of radial velocity averaged over the sensing volume \\
\hline$\sigma_{\mathrm{e}}^{2}=<V_{\mathrm{e}}^{2}>$ & Variance of random instrumental error of unbiased estimate of the radial velocity \\
\hline
\end{tabular}




\begin{tabular}{ll}
\hline$\sigma_{\mathrm{e}}$ & Instrumental error of radial velocity estimate \\
$\sigma_{\mathrm{L}}^{2}=<V_{\mathrm{L}}^{2}>-<V_{\mathrm{L}}>^{2}$ & Variance of the lidar estimate of radial velocity \\
$\sigma_{\mathrm{p}}$ & Duration of the probing pulse determined by the $e^{-1}$ power level \\
$\sigma_{\mathrm{r}}^{2}=<V_{\mathrm{r}}^{2}>-<V_{\mathrm{r}}>^{2}$ & Variance of the radial velocity \\
$\bar{\sigma}_{\mathrm{r}}^{2}$ & Variance of the radial velocity after averaging over azimuth angle interval $\left[0,360^{\circ}\right]$ \\
$\sigma_{\mathrm{t}}^{2}=\sigma_{\mathrm{r}}^{2}-\sigma_{\mathrm{a}}^{2}$ & Turbulent broadening of the Doppler spectrum \\
$\sigma_{u}^{2}=<u^{2}>$ & Variance of longitudinal wind component \\
$\sigma_{v}^{2}=<v^{2}>$ & Variance of transverse wind component \\
$\sigma_{w}^{2}=<w^{2}>$ & Variance of vertical wind component \\
$\bar{\sigma}_{\alpha}^{2}=M^{-1} \sum_{m=0}^{M-1} \sigma_{\alpha}^{2}\left(\theta_{m}\right)$ & Variance $\sigma_{\alpha}^{2}$ averaged over azimuth angle interval $\left[0,360^{\circ}\right]$, where subscripts $\alpha$ means $L$ or $a$ \\
$\varphi$ & Elevation angle \\
$\varphi_{\mathrm{E}}$ & Elevation angle equal to 35.3 \\
$\omega_{\mathrm{s}}$ & Angular rate of conical scanning \\
\hline
\end{tabular}


Competing interests. The authors declare that they have no conflict of interest.

Acknowledgements. The authors are grateful to colleagues from the Institute of Atmospheric Optics SB RAS - A. V. Falits, Yu. A. Rudi, and E. V. Gordeev - for their help in the experiments.

This study was supported by the Russian Science Foundation, project no. 14-17-00386-II.

Edited by: Ulla Wandinger

Reviewed by: two anonymous referees

\section{References}

Banakh, V. and Smalikho, I.: Coherent Doppler Wind Lidars in a Turbulent Atmosphere, Artech House Publishers, Boston, London, 2013.

Banakh, V. A. and Smalikho, I. N.: Lidar observations of atmospheric internal waves in the boundary layer of atmosphere on the coast of Lake Baikal, Atmos. Meas. Tech., 9, 5239-5248, https://doi.org/10.5194/amt-9-5239-2016, 2016.

Banakh, V. A., Smalikho, I. N., Falits, A. V., Belan, B. D., Arshinov, M. Y., and Antokhin, P. N.: Joint radiosonde and Doppler lidar measurements of wind in the boundary layer of the atmosphere, Atmos. Ocean. Opt., 28, 185-191, https://doi.org/10.1134/S1024856015020025, 2015.

Banta, R. M., Pichugina, Y. L., and Brewer, W. A.: Turbulent velocity-variance profiles in the stable boundary layer generated by a nocturnal low-level jet, J. Atmos. Sci., 63, 2700-2719, https://doi.org/10.1175/JAS3776.1, 2006.

Byzova, N. L., Ivanov, V. N., and Garger, E. K.: Turbulence in Atmospheric Boundary Layer, Gidrometeoizdat, Leningrad, 265 pp., 1989.

Davies, F., Collier, C. G., Pearson, G. N., and Bozier, K. E.: Doppler lidar measurements of turbulent structure function over an urban area, J. Atmos. Ocean. Tech., 21, 753-761, https://doi.org/10.1175/15200426(2004)021<0753:DLMOTS>2.0.CO;2, 2004.

Eberhard, W. L., Cupp, R. E., and Healy, K. R.: Doppler lidar measurement of profiles of turbulence and momentum flux, J. Atmos. Ocean. Tech., 6, 809-819, https://doi.org/10.1175/15200426(1989)006< 0820:AOADLM>2.0.CO;2, 1989.

Frehlich, R. G. and Cornman, L. B.: Estimating spatial velocity statistics with coherent Doppler lidar, J. Atmos. Ocean. Tech., 19, 355-366, https://doi.org/10.1175/1520-0426-19.3.355, 2002.

Frehlich, R. G., Hannon, S. M., and Henderson, S. W.: Coherent Doppler lidar measurements of wind field statistics, Bound.-Lay. Meteorol., 86, 223-256, https://doi.org/10.1023/A:1000676021745, 1998.

Frehlich, R. G., Meillier, Y., Jensen, M. L., Balsley, B., and Sharman, R.: Measurements of boundary layer profiles in urban environment, J. Appl. Meteorol. Clim., 45, 821-837, https://doi.org/10.1175/JAM2368.1, 2006.
Gal-Chen, T., Xu, M., and Eberhard, W. L.: Estimations of atmospheric boundary layer fluxes and other turbulence parameters from Doppler lidar data, J. Geophys. Res. 97, 409-418, https://doi.org/10.1029/91JD03174, 1992.

Kolmogorov, A. N.: Local structure of turbulence in incompressible viscous fluid at very large Reynolds numbers, Doklady AN SSSR, 30, 299-303, 1941.

Lumley, J. L. and Panofsky, H. A.: The Structure of Atmospheric Turbulence, New York (Interscience Publishers), 1964.

Monin, A. S. and Yaglom, A. M.: Statistical Fluid Mechanics, in: Volume II: Mechanics of Turbulence, M.I.T. Press, Cambridge, Mass., 1971.

O'Connor, E. J., Illingworth, A. J., Brooks, I. M., Westbrook, C. D., Hogan R. J., Davies, F., and Brooks, B. J.: A method for estimating the kinetic energy dissipation rate from a vertically pointing Doppler lidar, and independent evaluation from balloonborne in situ measurements, J. Atmos. Ocean. Tech., 27, 16521664, https://doi.org/10.1175/2010JTECHA1455.1, 2010.

Pearson, G., Davies, F., and Collier, C.: An analysis of performance of the UFAM pulsed Doppler lidar for the observing the boundary layer, J. Atmos. Ocean. Tech., 26, 240-250, https://doi.org/10.1175/2008JTECHA1128.1, 2009.

Sathe, A. and Mann, J.: A review of turbulence measurements using ground-based wind lidars, Atmos. Meas. Tech., 6, 3147-3167, https://doi.org/10.5194/amt-6-3147-2013, 2013.

Sathe, A., Mann, J., Vasiljevic, N., and Lea, G.: A six-beam method to measure turbulence statistics using ground-based wind lidars, Atmos. Meas. Tech., 8, 729-740, https://doi.org/10.5194/amt-8729-2015, 2015

Smalikho, I. N. and Banakh, V. A.: Accuracy of estimation of the turbulent energy dissipation rate from wind measurements with a conically scanning pulsed coherent Doppler lidar. Part I. Algorithm of data processing, Atmos. Ocean. Opt., 26, 404-410, https://doi.org/10.1134/S102485601305014X, 2013.

Smalikho, I. N. and Banakh V. A.: Investigation of feasibility of wind turbulence measurement by a pulsed coherent Doppler lidar in the atmospheric boundary layer, in: Proceedings of 28th International Laser Radar Conference, 25-30 June 2017, Bucharest, Romania, 2017.

Smalikho, I., Köpp, F., and Rahm, S.: Measurement of atmospheric turbulence by 2-micron Doppler lidar, J. Atmos. Ocean. Tech., 22, 1733-1747, https://doi.org/10.1175/JTECH1815.1, 2005.

Smalikho, I. N. Banakh, V. A., Pichugina, Y. L., and Brewer, W. A.: Accuracy of estimation of the turbulent energy dissipation rate from wind measurements with a conically scanning pulsed coherent Doppler lidar. Part II. Numerical and atmospheric experiments, Atmos. Ocean. Opt., 26, 411-416, https://doi.org/10.1134/S1024856013050151, 2013.

Vinnichenko, N. K., Pinus, N. Z., Shmeter, S. M., and Shur G. N.: Turbulence in the Free Atmosphere, edited by: Dutton, J. A., Consultants Bureau, New York, 262 pp., 1973. 